\title{
Distinctive Nuclear Localization Signals in the Oomycete Phytophthora sojae
}

\author{
Yufeng Fang ${ }^{1,2}$, Hyo Sang Jang ${ }^{3}$, Gregory W. Watson ${ }^{4,5}$, Dulani P. Wellappili ${ }^{2}$ and \\ Brett M. Tyler ${ }^{1,2 *}$ \\ 1 Interdisciplinary Ph.D. Program in Genetics, Bioinformatics and Computational Biology, Virginia Tech, Blacksburg, VA, USA \\ ${ }^{2}$ Center for Genome Research and Biocomputing and Department of Botany and Plant Pathology, Oregon State University, \\ Corvallis, OR, USA, ${ }^{3}$ Department of Environmental and Molecular Toxicology, Oregon State University, Corvallis, OR, USA, \\ ${ }^{4}$ Molecular and Cellular Biology Program, Oregon State University, Corvallis, OR, USA, ${ }^{5}$ Biological and Population Health \\ Sciences, Oregon State University, Corvallis, OR, USA
}

To date, nuclear localization signals (NLSs) that target proteins to nuclei in oomycetes have not been defined, but have been assumed to be the same as in higher eukaryotes. Here, we use the soybean pathogen Phytophthora sojae as a model to investigate these sequences in oomycetes. By establishing a reliable in vivo NLS assay based on confocal microscopy, we found that many canonical monopartite and bipartite classical NLSs (cNLSs) mediated nuclear import poorly in P. sojae. We found that efficient localization of $P$. sojae nuclear proteins by cNLSs requires additional basic amino acids at distal sites or collaboration with other NLSs. We found that several representatives of another well-characterized NLS, proline-tyrosine NLS (PY-NLS) also functioned poorly in P. sojae. To characterize PY-NLSs in $P$. sojae, we experimentally defined the residues required by functional PY-NLSs in three $P$. sojae nuclear-localized proteins. These results showed that functional $P$. sojae PY-NLSs include an additional cluster of basic residues for efficient nuclear import. Finally, analysis of several highly conserved $P$. sojae nuclear proteins including ribosomal proteins and core histones revealed that these proteins exhibit a similar but stronger set of sequence requirements for nuclear targeting compared with their orthologs in mammals or yeast.

Keywords: oomycetes, Phytophthora sojae, nuclear localization, nucleus labeling, NLS, classical NLS, PY-NLS

\section{INTRODUCTION}

In eukaryotes, many proteins such as core histones, transcription factors, and ribosomal proteins must be transported into the nucleus to accomplish their functions. Transport of those proteins into the nucleus occurs through large, proteinaceous structures called nuclear pore complexes (NPCs). Generally, NPCs allow passive diffusion of molecules smaller than $40-60 \mathrm{kDa}$, but require an appropriate sorting signal for passage of larger proteins (Lange et al., 2007). The sorting signals carried by those proteins are called nuclear localization signals (NLSs), that generally are short stretches of amino acids recognized by nucleo-cytoplasmic transporters (karyopherins) that promote active transport of proteins into the nucleus (Xu et al., 2010; Marfori et al., 2011). To date, two principal types of NLSs have been defined: the classical NLS (cNLS) and the proline-tyrosine NLS (PY-NLS). 
cNLSs are the best-characterized nuclear targeting signals, and are recognized by karyopherin- $\beta 1$ (Importin- $\beta$, Kap $\beta 1$ ) through direct binding to an adaptor protein karyopherin- $\alpha$ (Importin- $\alpha, \mathrm{Kap} \alpha$ ) (Lange et al., 2007; Marfori et al., 2011). According to the numbers of basic amino acid clusters within them, cNLSs are further divided into two subclasses, monopartite, and bipartite cNLSs. Monopartite cNLSs contain a single stretch of basic amino acids which may consist of at least four consecutive basic amino acids, exemplified by the SV40 large $\mathrm{T}$ antigen NLS (PKKKRKV) (Kalderon et al., 1983, 1984). Alternatively, three non-consecutive basic amino acids may suffice, exemplified by the $c-M y c$ protooncoprotein NLS (PAAKRVKLD) (Makkerh et al., 1996). Traditionally, the monopartite cNLS has a consensus of $\mathrm{K}(\mathrm{K} / \mathrm{R}) \mathrm{X}(\mathrm{K} / \mathrm{R})$ (Lange et al., 2007). Bipartite cNLSs have two stretches of basic amino acids separated by $10-12$ amino acids (Lange et al., 2007). They were first found in Xenopus nucleoplasmin (KRPAATKKAGQAKKKK) (Dingwall et al., 1982) and are represented by the consensus sequence $(\mathrm{K} / \mathrm{R})(\mathrm{K} / \mathrm{R}) \mathrm{X}_{10-12}(\mathrm{~K} / \mathrm{R})_{3 / 5}\left(\mathrm{X}\right.$ is any amino acid and $(\mathrm{K} / \mathrm{R})_{3 / 5}$ represents three lysine or arginine residues out of five consecutive amino acids) (Dingwall and Laskey, 1991). With increasing numbers of cNLS-bearing proteins identified, and more indepth biochemical and biophysical analyses, the cNLS consensus sequences have been progressively expanded (reviewed in Marfori et al., 2011).

The PY-NLS is recognized for nuclear import by karyopherin$\beta 2(\operatorname{Kap} \beta 2)$ in humans and by its ortholog Kap104 in yeast (Lee et al., 2006). Compared to the cNLS, fewer PY-NLS proteins have been characterized experimentally $(\sim 42$ through 2015 , Soniat and Chook, 2015). PY-NLSs are generally longer (1530 residues) and more variable than cNLSs, making it more difficult to clearly define their common features (Xu et al., 2010; Chook and Suel, 2011). M9NLS is the best-characterized PY-NLS. It consists of a 38-residue domain from a splicing factor, heterogeneous nuclear ribonucleoprotein A1 (hnRNP A1) (Bonifaci et al., 1997; Truant et al., 1999). On the basis of the crystal structure of human $\operatorname{Kap} \beta 2$ bound to M9NLS, Lee et al. (2006) proposed three rules for PY-NLSs: (1) structurally disordered in free substrates; (2) overall basic character; and (3) central hydrophobic or basic motif (epitope 1) followed by the motif $\mathrm{R} / \mathrm{H} / \mathrm{K}-\mathrm{X}_{2-5}-\mathrm{PY}$ (the $\mathrm{R} / \mathrm{H} / \mathrm{K}$ - and PY-motifs are defined as epitopes 2 and 3 respectively). According to the composition of the amino acids in epitope 1, PY-NLSs have been further classified into hydrophobic and basic PY-NLSs (hPYand bPY-NLSs) (Lee et al., 2006). Later, the rules were updated to accommodate additional features identified in Saccharomyces cerevisiae: the Kap $\beta 2$ ortholog Kap104 only recognizes the basic but not the hydrophobic PY-NLS (Süel et al., 2008); and the tyrosine in the C-terminal PY epitope (epitope 3) displays degeneracy; not only PY but also some other motifs like PL could be recognized by yeast Kap104 (Süel et al., 2008). In silico predictions suggest that Kap $\beta 2$-mediated import accounts for a substantial fraction of substrates involved in RNA processing and transcription factors (Süel and Chook, 2009; Chook and Suel, 2011).
Based on a growing volume of functional data from human and yeast nuclear translocation signals, a number of prediction software packages have been developed to bioinformatically predict nuclear-localized proteins and the responsible signals. Those tools can be classified into two general groups, namely classification of subcellular or nuclear localization; and detection of NLS sequences (Marfori et al., 2011). Software such as WoLF PSORT (Horton et al., 2007) and NucPred can be used for predicting if proteins of interest have nuclear localization based on the general properties of the proteins, while PSORT II (PSORT, 1997), NLStradamus (Nguyen Ba et al., 2009), and cNLS Mapper (Kosugi et al., 2009) predict residues that may serve as NLSs. PredictNLS (Cokol et al., 2000) provides prediction for both subcellular localization and the NLS sequences of given proteins. Of those NLS predictors, PSORT II and its extension WoLF PSORT are the two most widely adopted tools that are built on NLS consensus sequences (PSORT, 1997; Horton et al., 2007). In contrast, NLStradamus is based on experimentally validated NLSs in yeast (Nguyen Ba et al., 2009), and cNLS Mapper is based on synthetic peptide library data (Kosugi et al., 2009). Most of the computational tools to date are designed for prediction of cNLS, except NLStradamus, which does not distinguish the NLS types.

Phytophthora sojae is a destructive oomycete pathogen that infects soybean seedlings as well as established plants (Tyler, 2007). Although oomycetes physiologically and morphologically resemble fungi, molecular taxonomy has shown that oomycetes are phylogenetically close to algae and diatoms (Tyler, 2007; Kamoun et al., 2015). Oomycetes are diploid and lack a free haploid life stage. The genomes of oomycetes (50-250 Mb) are also generally larger than those of true fungi (10-40 Mb) (reviewed in Judelson and Blanco, 2005). Most Phytophthora species are plant pathogens and together damage a huge range of agriculturally and ornamentally important plants (Erwin and Ribeiro, 1996). For instance, $P$. infestans, causes the potato lateblight disease that resulted in the Irish potato famine, and continues to be a problem for potato and tomato crops (Judelson and Blanco, 2005). P. sojae causes around \$1-2 billion in losses per year to the soybean crop (Tyler, 2007). Because of its economic impact, $P$. sojae, along with $P$. infestans, has been developed as a model species for the study of oomycete plant pathogens (Tyler, 2007).

To date, most nuclear localization studies have been carried out in model organisms, and no NLS sequences have yet been defined in oomycetes. Here we find that many eukaryotic NLS sequences function poorly if at all in $P$. sojae. We demonstrate that efficient localization of $P$. sojae nuclear protein by cNLSs requires additional basic amino acids at distal sites or collaboration with other NLSs. Furthermore, we show that a fully functional PY-NLS requires additional basic residues either within the motif itself or adjacent to the motif. Finally, comparison of the nuclear localization activities of NLS sequences from $P$. sojae ribosomal proteins and core histones with those from other eukaryotes reveals that $P$. sojae may use modified nuclear import mechanisms for those highly conserved nuclear proteins. 


\section{MATERIALS AND METHODS}

\section{$P$. sojae Strains and Growth Conditions}

All of the NLS tests were carried out in the $P$. sojae reference isolate $\mathrm{P} 6497$ (race 2). Cultures were routinely grown and maintained in cleared $\mathrm{V} 8$ medium at $25^{\circ} \mathrm{C}$ in the dark. $P$. sojae transformants were incubated in 12-well plates containing liquid V8 media supplemented with $50 \mu \mathrm{g} / \mathrm{ml}$ G418 (Geneticin) for 2-3 days before examination by confocal microscopy.

\section{Sequence Analysis}

Phytophthora protein sequences and IDs were obtained from FungiDB (Stajich et al., 2012). Their corresponding accession numbers in the GenBank database are listed in Table S1. Ribosomal protein and core histone sequences of Arabidopsis, yeast and human were obtained from the GenBank database: Arabidopsis L27a (accession number, NP_177217), H3.1 (NP_201339), H4, (NP_190179); human H3.k (P68431), H4.j (AAA52652), L27a (NP_000981); Saccharomyces cerevisiae L28 (NP_011412), H3 (or Hht2p, CAY82162); H4 (or Hhf2p, NP_014368). Protein sequence alignments were carried out using Clustal Omega (Sievers et al., 2011). Additional sequence information for the proteins PHYSO_357835, PHYSO_480605, PHYSO_251824, PHYSO_561151, and PHYSO_533817 can be found in the Data Sheet 1 (Supplemental Sequences).

\section{Construction of Plasmids}

All the primers used in this study are listed in Table S2 in the Supplementary Material. All the fusion protein reporter constructs in which the NLS was fused to the N-terminus of GFP or 2XGFP were based on the plasmid backbone pYF2-2XGFP (Fang and Tyler, 2016). To test reporters with the NLS fused to the C-terminus of GFP, a new plasmid backbone pYF3-2XGFP was generated by inserting an $e G F P$ fragment with attached Cterminal multiple cloning sites and stop codon (Bsr GI-Hpa I-Bsp EI-Mlu I-TAA) into the Afl II and Apa I sites of the backbone pYF2-GFP. To clone NLS candidates efficiently and economically, NLSs derived from exogenous genes smaller than $\sim 80$ bp were created by oligo annealing and inserted into the Spe I and Sac II sites of pYF2-2XGFP, or the Bsr GI and Bsp EI sites of pYF3-2XGFP. PCR products from $P$. sojae genes were inserted into the Stu I site of pYF2-2XGFP or the Hpa I site of pYF3-2XGFP by blunt ligation. The $P$. sojae $\mathrm{H} 2 \mathrm{~B}$ nuclear marker fusions (H2B-GFP and $\mathrm{H} 2 \mathrm{~B}$-mCherry) and the $P$. capsici fibrillarin nucleolar marker fusion (FIB-mCherry) were created using the pGFPN or pMCherryN plasmids (Ah-Fong and Judelson, 2011). Point mutations and deletions of DNA sequences were made through QuikChange Lightning Multi SiteDirected Mutagenesis (Agilent Technologies).

To express 2XGFP fusions in mammalian cells, genes encoding 2XGFP and its SV40 NLS and M9NLS fusions were PCR-amplified from $P$. sojae expression plasmids using primers pYF2_GW_F and GFP_stop_GW_R, and integrated into pcDNA 3.2/V5-DEST by Gateway ${ }^{\mathrm{TM}}$ cloning (Thermo Scientific). Integration of 2XGFP into pcDNA 3.2/V5-DEST also generated a plasmid backbone pcDNA 3.2-2XGFP, in which a preserved
Eco RV site originating from the pYF2 backbone was used for insertion of PY-NLS amplicons by blunt ligation.

PCR-amplification was conducted using Phusion $^{\circledR}$ High-Fidelity DNA Polymerase (NEB). Standard molecular techniques were performed as described by Sambrook and Russell (2001) or according to instructions from kit manufacturers.

\section{$P$. sojae Transformation}

Polyethylene glycol (PEG)-mediated protoplast transformations were conducted by an improved protocol as described previously (Fang and Tyler, 2016). In general, P. sojae protoplasts were isolated by enzyme digestion using 0.5\% Lysing Enzymes from Trichoderma harzianum (Sigma L1412) and 0.5\% CELLULYSIN $^{\circledR}$ Cellulase (Calbiochem 219466) in $0.4 \mathrm{M}$ mannitol, $20 \mathrm{mM} \mathrm{KCl,} 20 \mathrm{mM}$ MES, pH 5.7, $10 \mathrm{mM} \mathrm{CaCl}_{2}$. One milliliter of protoplasts at a density of $2 \times 10^{6}-2 \times 10^{7} / \mathrm{ml}$ and 20-30 $\mu \mathrm{g}$ DNA were used for single plasmid transformations. For co-transformation experiments, 20-30 $\mu \mathrm{g}$ of the plasmid carrying the NPT II selectable marker gene was used, together with an equimolar ratio of any other DNAs included. Protoplasts were regenerated overnight in regeneration media (pea broth containing $0.5 \mathrm{M}$ mannitol). Regenerated protoplasts were collected and grown as colonies in solid regeneration media containing $50 \mu \mathrm{g} / \mathrm{ml} \mathrm{G} 418$ (Geneticin) for 2 days. G418-resistant colonies were transferred to 12 -well plates containing liquid V8 media supplemented with $50 \mu \mathrm{g} / \mathrm{ml} \mathrm{G} 418$ and incubated for 2-3 days at $25^{\circ} \mathrm{C}$ before confocal microscopy observation. Under these conditions, $P$. sojae transformant colonies typically continue to express genes introduced on exogenous DNA for 1-3 weeks, even if the genes are not integrated into the chromosomes. Here we did not test whether the exogenous DNA had integrated in the chromosomes; thus we refer to the mycelial colonies recovered by our procedure as transient transformants.

\section{Human Cell Line Transfection and Immunocytochemistry}

HEK293 cells were maintained in DMEM media (Mediatech) supplemented with $10 \%$ fetal bovine serum (Tissue Culture Biologicals), penicillin-streptomycin solution ( $1 \mathrm{IU} / \mathrm{ml}$ penicillin and $100 \mathrm{ug} / \mathrm{ml}$ streptomycin, Mediatech). Transfection was done according to the manufacturer's protocol. Briefly, HEK293 cells were plated on a glass coverslip in a 24-well plate (Greiner Bio-One) at a density sufficient for $70 \%$ confluency at the time of transfection. Cells were transfected with $500 \mathrm{ng}$ DNA and 1 $\mu \mathrm{l}$ of Lipofectamine 2000 (Invitrogen) per well for $48 \mathrm{~h}$. For immunocytochemistry, the transfected cells were fixed with $3.7 \%$ $(\mathrm{w} / \mathrm{v})$ paraformaldehyde in PBS for $15 \mathrm{~min}$ at RT. The fixed cells were permeabilized with $0.1 \%$ Triton X-100 in PBS for $10 \mathrm{~min}$ at RT and incubated with $1 \%$ bovine serum albumin in PBS for $1 \mathrm{~h}$ at RT. Then, the cells were incubated with rabbit anti-GFP antibody conjugated with AlexaFluor 488 (Invitrogen) overnight at $4{ }^{\circ} \mathrm{C}$. Cells on the coverslip were mounted on a glass slide using ProLong Gold antifade with DAPI (Invitrogen) and dried overnight at RT in darkness. 


\section{Confocal Microscopy Imaging}

Laser scanning confocal microscopy (Zeiss LSM 780 NLO) was used to monitor the subcellular localization of fluorescent protein fusions in $P$. sojae and human cell transformants. Clumps of 2-3 days old transformed $P$. sojae mycelia grown in liquid V8 media were removed with a toothpick, washed, and maintained in modified Plich media $\left(0.5 \mathrm{~g} \mathrm{KH}_{2} \mathrm{PO}_{4}, 0.25 \mathrm{~g} \mathrm{MgSO}_{4} \bullet 7 \mathrm{H}_{2} \mathrm{O}, 1 \mathrm{~g}\right.$ asparagine, $1 \mathrm{mg}$ thiamine, 0.5 g yeast extract, $10 \mathrm{mg} \beta$-sitosterol, 25 g glucose dissolved in 11 water) before observation. For DAPI staining, mycelia samples were pre-treated with Phosphatebuffered saline (PBS, pH 7.4) containing $0.2 \mu \mathrm{g} / \mathrm{ml}$ DAPI for 25 min in dark, and washed twice with PBS according to Hardham (2001). Images were captured using a $63 \mathrm{X}$ oil objective with excitation/emission settings (in $\mathrm{nm}$ ) of 405/410-490 for DAPI, 488/504-550 for GFP, and 561/605-650 for mCherry. For each sample, at least three independent $P$. sojae transformants were examined. Images were adjusted using the microscope's builtin Zen 2012 software (Blue and/or Black edition according to different purposes). Images were cropped, and the tonal range was increased by adjusting highlights and shadows without altering the color balance.

To quantitate the ratio of nuclear to cytoplasmic localization, nuclear and cytoplasmic intensities were collected by manually defining the nuclear and cytoplasmic regions in each image as described in Hunter et al. (2014). The mean intensity values of the defined regions were generated by the Zen 2012 (Blue edition) "measure" tool, which ignores any adjustments of tonal range. In most cases, nuclei were identified by the morphology of the GFP-stained region (a large, uniformly stained, irregular ovoid region, often with an unstained nucleolus), and were not confirmed by co-expression of $\mathrm{H} 2 \mathrm{~B}$-mCherry. Where nuclei could not be identified due to very poor nuclear localization, $\mathrm{H} 2 \mathrm{~B}-\mathrm{mCherry}$ was co-expressed to verify the nuclei. The nuclear to cytoplasmic fluorescence ratio was calculated using the means ( \pm standard error) of the $\log _{2}$ transformed ratios from 30 pairs of adjacent nuclear and cytoplasmic regions, chosen at random from each of three independent $P$. sojae transformants. This procedure produced the $\log _{2}$ (nuclear to cytoplasmic ratio) or LNC used to characterize the nuclear localization activity of most constructs in this study. Significance was tested with two-sample, unpaired $t$-tests of the LNC-values performed by the software GraphPad Prism 7, and a false discovery rate (FDR)-adjusted $p^{\prime}$ value (Benjamini and Hochberg, 1995) of 0.001-0.05, as noted in each figure legend.

\section{RESULTS}

\section{Establishment of Reliable Fluorescent Labeling of $P$. sojae Nuclei for Assay of Nuclear Localization}

To assess the activity of NLSs, we implemented a classic in vivo NLS assay, in which a candidate NLS was fused to GFP or to two fused GFP moieties (2XGFP) and then transiently expressed in individual $P$. sojae transformants. The fusion of two GFP molecules created a protein $(\sim 55 \mathrm{kDa})$ larger than the NPC threshold for passive diffusion. Subcellular localization of the proteins was visualized by live-cell imaging in at least three individual transformant colonies per construct, using confocal laser scanning microscopy. To assist in verifying the nuclear localization of a protein, the commonly used nuclear dye DAPI (4', 6-diamidino-2-phenylindole) was initially employed, because it had been reported to label the nuclei of living Hardham, 2001; Zhang et al., 2012 or fixed (Gamboa-Mélendez et al., 2013) Phytophthora tissues. However, when living $P$. sojae hyphae were stained with DAPI, the NLS-fused GFPs were extensively distributed into the cytoplasm (Figure S1A). This contrasted with the clear nuclear localization observed in hyphae that were not stained with DAPI (Figure S1A). In particular, when using a $2 \mathrm{XGFP}$ reporter, fused to a strong synthetic NLS, PsNLS (Fang and Tyler, 2016), we noticed that regions of hyphae with poor DAPI staining exhibited strong GFP nuclear localization while regions of hyphae well-stained with DAPI showed poor GFP nuclear localization (Figure S1B). This suggested that DAPI staining caused mis-localization of nuclear-localized proteins to the cytoplasm. Indeed, a timelapse experiment tracking a hypha during DAPI staining showed that after $18 \mathrm{~min}$ staining, the nuclei disintegrated and the nuclear-localized PsNLS-2XGFP was released into the cytoplasm (Figure S1C and Supplemental Video 1).

To identify a reliable strategy to label $P$. sojae nuclei, we tested other nuclear staining dyes, such as Hoechst 33342. However, $P$. sojae hyphae were not permeable to that dye (data not shown). We also tried to stain the nuclei of fixed hyphae using DAPI according to a protocol described by Gamboa-Mélendez et al. (2013), but redistribution of nuclear GFP into the cytoplasm was still commonly observed. Therefore, we sought to label $P$. sojae nuclei by expressing a nuclear-targeted fluorescent protein. P. sojae core histone $\mathrm{H} 2 \mathrm{~B}$ fused to mCherry was selected according to Ah-Fong and Judelson (2011), and this fusion showed predominant nuclear localization in $P$. sojae hyphae (Figure 1A). Expression of H2B-mCherry appeared to cause some toxicity to $P$. sojae, because reduced numbers of transiently transformed $P$. sojae colonies were obtained with this construct. Similar results were observed with a $P$. sojae histone $\mathrm{H} 1$ fusion protein, and even worse with histone $\mathrm{H} 3$ and $\mathrm{H} 4$ fusions (data not shown). Thus, we settled on $\mathrm{H} 2 \mathrm{~B}$-mCherry as a nuclear marker for co-expression with NLS-GFP reporter genes.

\section{Monopartite cNLSs Show Weak Nuclear Targeting Activity in $P$. sojae}

To examine cNLS activity in $P$. sojae, three well-characterized monopartite cNLS sequences were individually fused to the Nterminus of 2XGFP and expressed in P. sojae transformants. To quantify the activities of different NLSs, the ratio of fluorescence intensity in nuclei compared to the cytoplasm (Nuc:Cyt) was measured within $\sim 30$ hyphae from at least three individual $P$. sojae transformants for each fusion. Due to the wide range of nuclear-cytoplasmic ratios observed, we found it convenient to express these ratios as $\log _{2}$ (Nuc:Cyt), or LNC. As expected, 2XGFP alone was extensively localized to the cytoplasm (LNC $=0.52$; Figures 1A,D). The well-studied cNLS derived from SV40 large T antigen (SV40 NLS) produced incomplete albeit statistically significant (FDR $p^{\prime}<0.001$ ) nuclear localization 
A

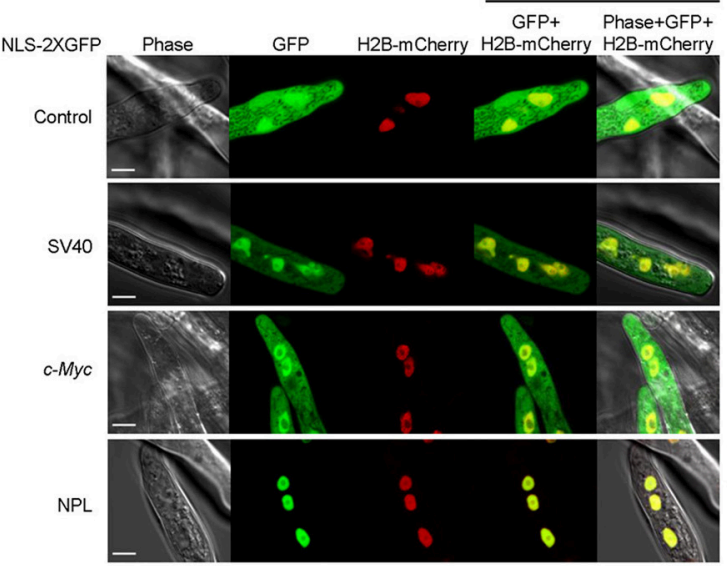

C

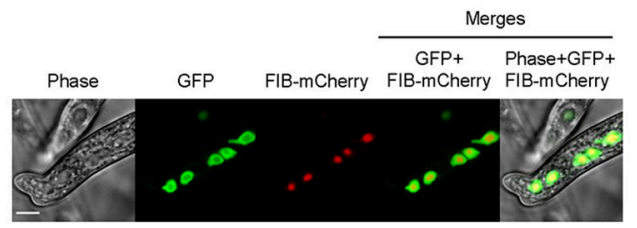

E

$[K / R] \underline{(K / R)(K / R)} X_{10-12}^{(K / R)} \underline{(15}_{3 / 5}$
B
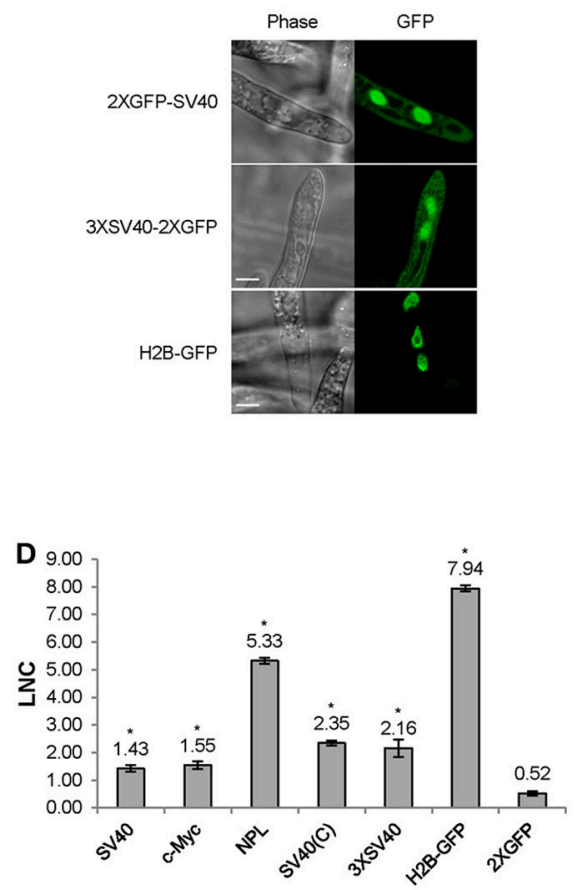

NPL (F) $155 \bullet$ (F) PHYSO_561151 (F) $504 \bullet$ (FKRRSTSGHP-GLSAKRNKtsmg 520 PHYSO 561151 (NF) $505 \bullet$ @RRSTSGHP-GLSAKRNKKtsm 521

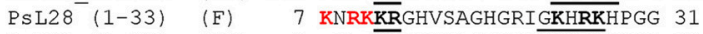
PsL28 (1-33) $\mathrm{m}$ (NF) 7 aNaa $\overline{\text { KR }}$ GHVAGHGRI GKHRKHPGG 31

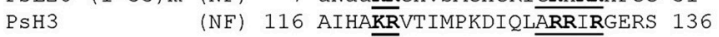

$\mathbf{F}$
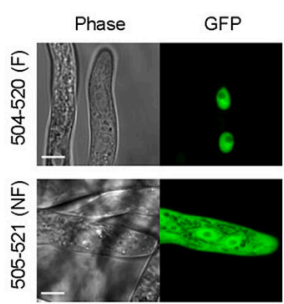

PHYSO_561151
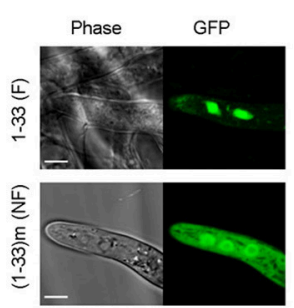

PsL28

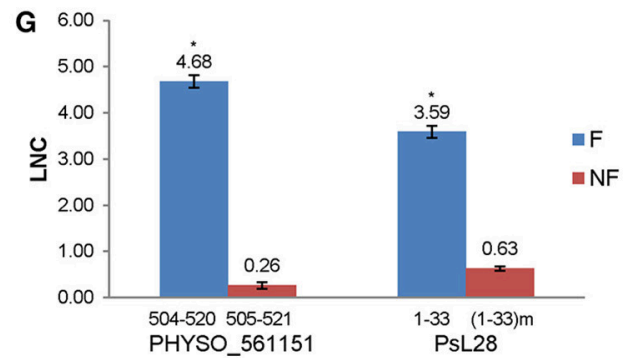

FIGURE 1 | Functional characterization of monopartite and bipartite cNLSs in $\boldsymbol{P}$, sojae transformants. (A) Subcellular localization of N-terminal cNLS-2XGFP fusions, involving mammalian cNLS sequences. SV40, c-Myc, NPL, represent cNLSs identified in SV40 large T antigen, human c-Myc proto-oncoprotein, and Xenopus nucleoplasmin respectively; H2B-mCherry = P. sojae histone H2B fused to the N-terminus of mCherry; Control = 2XGFP. "GFP" above the panel indicates visualization of the relevant GFP or 2xGFP fusions. Scale bar corresponds to $5 \mu \mathrm{m}$ in this and all subsequent figures. (B) Subcellular localization of different fusions with the SV40 NLS. H2B-GFP was used as a positive control. (C) Exclusion of NPL-2XGFP from the nucleolus, as confirmed by P. capsici fibrillarin fused to mCherry (FIB-mCherry). (D) Quantification of nuclear localization by protein segments shown in (A,B). For quantification in all figures, LNC indicates the mean log2-transformed nuclear fluorescence to cytoplasmic fluorescence ratio from $\sim 30$ randomly selected pairs of nuclear and adjacent cytoplasmic regions. SV40 (C) = SV40 NLS attached at the C-terminus of 2XGFP (i.e., 2XGFP-SV40 NLS). Error bars, S.E. Asterisks, LNC-values of NLS-GFP fusions that are significantly greater than the LNC of 2XGFP alone (FDR $\left.p^{\prime}<0.001\right)$. (E) Sequence alignment of predicted bipartite cNLSs tested in $P$. sojae. The proposed $P$. sojae bipartite cNLS consensus is shown on the top; $[K / R]$ in red indicates the position of additional positive residues required in $P$. sojae. In the sequences of each protein, the two elements of each bipartite CNLS predicted by PSORT // (PSORT, 1997) are underlined, and basic amino acids within each cluster are in bold. The additional basic amino acids demonstrated to contribute to nuclear localization are in bold red. The residues in lowercase indicate non-native residues flanking the candidate NLS in each construct. F, functional NLS; NF, non-functional NLS. For PHYSO_561151 and PSL28, both the full length functional cNLSs and the truncated or mutant non-functional cNLSs are shown. $\mathbf{( F , G ) ~ F u n c t i o n a l ~ t e s t s ~ o f ~} P$. sojae bipartite NLSs listed in $\mathbf{E}$. F, representative images. G, quantification. (1-33) $m$ indicates mutation of the red-highlighted residues in PsL28 (K7A/R9A/K10A). Representative images are shown in (A-C,F). 
in any fusion configuration (fusion at $\mathrm{N}$ - or C-terminus of 2XGFP) or copy number (LNC $=1.43-2.35$; Figures 1A,B,D). In comparison, H2B-GFP exhibited an LNC of 7.94. Another monopartite cNLS prototype, the $c-M y c$ NLS, produced similar results $(\mathrm{LNC}=1.55$; Figures $1 \mathrm{~A}, \mathrm{D})$. To characterize cNLSs in native $P$. sojae nuclear proteins, we also analyzed eight protein fragments that contained monopartite cNLS motifs predicted by PSORT II (PSORT, 1997). As summarized in Table S3, we observed that none of the predicted monopartite cNLSs were sufficient for nuclear localization of GFP reporters.

\section{Functional Bipartite cNLSs Require Additional Basic Amino Acids Compared to the Conventional Bipartite Consensus}

To examine the nuclear localization activities of bipartite cNLSs, the classic bipartite cNLS of nucleoplasmin (NPL) was assayed in $P$. sojae. This sequence produced strong nuclear localization $(\mathrm{LNC}=5.33$; Figures 1A,D). Small unstained regions in the centers of nuclei were validated as nucleoli by coexpression of the nucleolar marker, fibrillarin (from Phytophthora capsici; Genbank accession No. KY452016) fused to mCherry (Figure 1C). To explore the activity of bipartite cNLSs further, four protein segments that were predicted to contain bipartite cNLS sequences (by PSORT II) were identified from nuclearlocalized $P$. sojae proteins, and assayed in $P$. sojae transformants. Of these four, two ( $\mathrm{PsH}_{116-136}$ and $\mathrm{PHYSO} 561151_{505-521}$ ) were incapable of producing strong localization of GFP reporters into P. sojae nuclei (Table S3). On the other hand, two regions carrying predicted bipartite cNLSs showed strong activity, namely one at the C-terminus of PHYSO_561151 and one at the $\mathrm{N}$-terminus of PsL28. At the C-terminus of PHYSO_561151, the functional residues proved to be residues 504-520, displaced one position from the inactive predicted sequence at 505-521 (LNC $=4.68$ ). At the N-terminus of PsL28, the functional residues appeared to be residues 7-27 (LNC = 3.59) (Figures 1E,F). Sequence comparisons of the functional bipartite cNLS sequences in PsL28 and PHYSO_561151 revealed the presence of additional arginine or lysine residues in the first of the two basic amino acid clusters that comprise the bipartite motif, compared to the canonical consensus developed from mammalian and yeast proteins (highlighted in red in Figure 1E). Mutation of these additional basic amino acids from the PsL28 or PHYSO_561151 bipartite NLSs showed that these residues were essential for the activity of these NLSs (Figures 1F,G). The human NPL bipartite cNLS however lacked any additional positive residues in the first basic cluster, but was functional. This cNLS has four consecutive lysines in the second cluster and two additional lysines between the two clusters; one or both of these features may make this cNLS functional in P. sojae. Although PSORT II did not accurately predict the functional bipartite NLSs in $\mathrm{PsH} 3$ and PsL28, both NLStradamus and cNLS Mapper identified broader regions that encompassed those extended NLSs (Tables S3, S4). Both programs also correctly predicted that $\mathrm{PsH}_{116-136}$ would not be functional.

\section{Canonical PY-NLS Motifs Produce Weak Nuclear Localization Activity in $P$. sojae}

Although a number of PY-NLS sequences have been characterized in human and yeast proteins, few of them have been reported in other organisms including oomycetes. To examine the activity of PY-NLS sequences in oomycetes, four well-characterized human and yeast PY-NLSs (two basic, bPY-NLS; two hydrophobic, hPY-NLS) were fused to the $\mathrm{N}$-terminus of $2 \mathrm{XGFP}$ and their localization was examined in P. sojae transformants (Figure 2A). None of these PY-NLSs produced strong nuclear localization of the 2XGFP reporter (Figures 2B,C). Only the bPY-NLS in hnRNP M produced significantly more nuclear localization than 2XGFP alone (FDR $p^{\prime}<0.001$ ) (Figures 2B,C). We also tested a synthetic peptide, M9M, that is a chimera of the hnRNP A1 (M9) hPY-NLS and the hnRNP M bPY-NLS (Figure 2A). This peptide has high affinity to the Kap $\beta 2$ PY-NLS binding site in human and is usually used as a Kap $\beta 2$-specific inhibitor (Cansizoglu et al., 2007). In P. sojae this peptide produced significantly more nuclear localization than the four PY-NLS prototypes (FDR $p^{\prime}<0.001$ ), although the localization was still weaker than that produced by the SV40 NLS (Figures 2B,C).

Given that some prototypical PY-NLSs produced weak nuclear localization in $P$. sojae, we considered the possibility that a fully functional PY-NLS in P. sojae may require additional elements, as was the case for cNLSs. To search for functional PY-NLS sequences in $P$. sojae nuclear-localized proteins, we began by scanning the $P$. sojae proteome using the human/yeast PY-NLS consensus sequences (the "PL" rule was allowed for bPY-NLS, Figure 3A). Then we filtered for structural disorder and overall positive charge in the NLS. This two step scan revealed 25 proteins containing candidate hPY-NLSs and 200 containing candidate bPY-NLSs. Twelve candidates annotated as RNAprocessing proteins or transcription factors were selected for further analysis.

We also extended the consensus-sequence-based search by including the "PL" option for hPY-NLSs. The rationale for this was that it was reported that the tyrosine in the Cterminal PY motif shows degeneracy in yeast (Süel et al., 2008). However, since yeast Kap104 only recognizes bPY-NLS motifs, the degeneracy of tyrosine in hPY-NLS was not clear. Thus, we reasoned that the "PL" rule for the bPY-NLSs may also apply to hPY-NLSs. By adding the "PL-rule" in the hPY-NLS search (Figure 3A), we obtained another 82 hPY-NLS candidates having C-terminal "PL" motifs. One candidate (PHYSO_480605, annotated as an mRNA maturation protein) was selected for further analysis because the putative PY-NLS was the only predicted NLS in the protein (Figure 3B). Another candidate, PHYSO_251824, had already been selected for testing because it also contained a separate conventional PY-NLS motif.

To validate the subcellular localization of the proteins, the 13 full-length proteins were tagged with GFP and expressed in $P$. sojae transformants. Five candidates showed strong nuclear localization in $P$. sojae hyphae at steady state, including PHYSO_480605 (Figure 3B and Table 1). PHYSO_561151 and PHYSO_357835 also showed nucleolar localization (Figure 3B). 

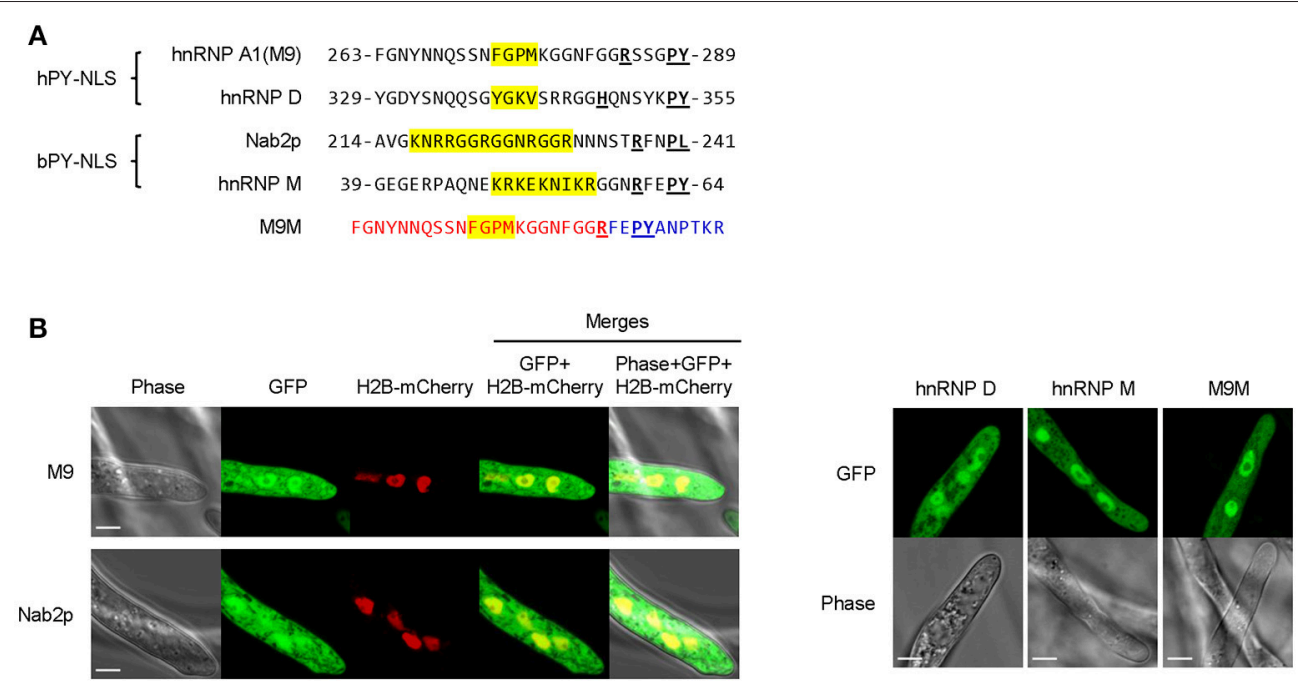

C

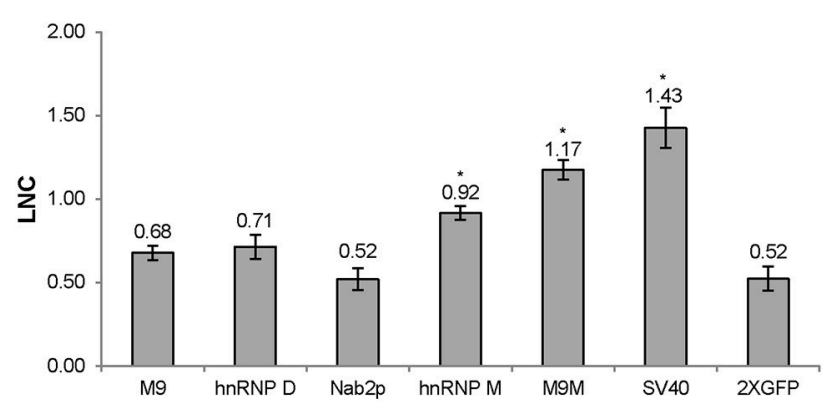

FIGURE 2 | PY-NLS prototypes exhibit weak nuclear targeting activities in $\boldsymbol{P}$. sojae transformants. (A) Sequences of four well-characterized PY-NLSs derived from human or yeast proteins and the Kap $\beta 2$-specific nuclear import inhibitor, M9M peptide. Core residues that determine hydrophobic or basic PY-NLS type are shaded in yellow. The R/K/H-X $2-5-P Y / L$ consensus residues are bold and underlined. Residues marked in red and blue in $\mathrm{M} 9 \mathrm{M}$ are sequences originating from M9 and hnRNP M respectively. (B) Subcellular localization of the five PY-NLS described in (A). Representative images are shown. (C) Quantification of localization of fusions observed in (B). For ease of comparison, the LNC-values of SV40 NLS-2XGFP and 2XGFP alone (from Figure 1) are included in the bar chart here and in subsequent figures; i.e., the same values were used in every figure. Asterisks, LNC-values of NLS-2XGFP fusions that are significantly greater (FDR $p^{\prime}<0.001$ ) than the LNC of 2XGFP.

(Although PHYSO_561151 was initially selected as a PYNLS candidate, its predicted PY-NLS proved to be inactive, as described below, and we subsequently determined that it contained a functional bipartite cNLS, as described above). Other candidates either could not be PCR amplified, contained incorrect intron annotations causing frame shifts, or appeared toxic when overexpressed in $P$. sojae transformants (data not shown).

To determine whether the predicted PY-NLSs in the nuclear-localized proteins were sufficient to mediate nuclear localization of reporter proteins, protein segments containing the motifs were fused to 2XGFP at either the $\mathrm{N}$ - or Cterminus, based on their positions in the native proteins. One PY-NLS-containing segment, PHYSO_357835338-387, mediated very efficient nuclear localization (Figure 3C). Other candidates, namely PHYSO_4806051-32 and PHYSO_251824239-358, produced incomplete nuclear localization with some remaining cytoplasmic signals (Figure 3C). In contrast, the PY-NLS-containing segments PHYSO_561151225-445 and
PHYSO_533817314-463 did not produce any nuclear localization (Figure 3C, Figures S2, S3). As noted above, nuclear localization of PHYSO_561151 subsequently turned out to be mediated by a bipartite cNLS at its C-terminus, while the nuclear localization of PHYSO_533817 was determined by an unidentified sequence between residues 172-314 (Figure S3).

Because four of the protein segments carrying candidate PYNLSs showed weak or non-existent nuclear localization activity in P. sojae, it was unclear if the problem was the general reliability of the three PY-NLS prediction rules or whether the P. sojae import machinery did not efficiently utilize the predicted PYNLS motifs. To address this question, parallel experiments were carried out in human embryonic kidney 293 cells (HEK 293) to determine the activity of the putative PY-NLSs in human cells. As shown in Figures 3C,D, the subcellular localization of the PY-NLS-containing protein segments in HEK 293 cells were well correlated with their localizations in $P$. sojae, except for segment PHYSO_4806051-32 that showed much stronger nuclear localization in the human cells. 


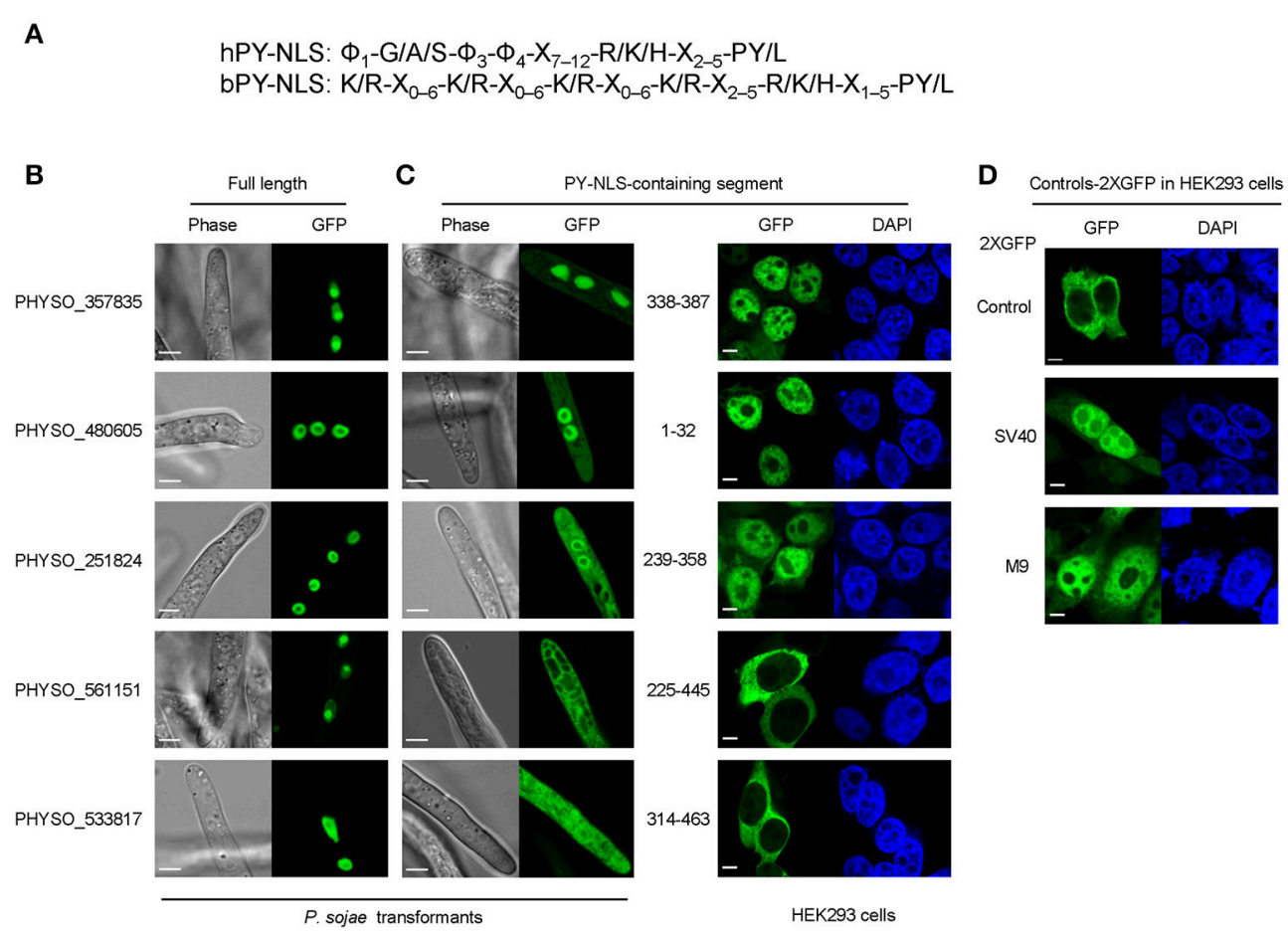

FIGURE 3 | Subcellular localization produced by five protein segments containing candidate PY-NLS motifs. (A) The modified consensus sequence (Süel et al., 2008) used for searching for candidate PY-NLSs in P. sojae. $\Phi 1$ represents a hydrophobic residue (defined here as L, M, I, V, W, Y, H, A, P, or F) while $\Phi 3$ and $\Phi 4$ represent either hydrophobic residues or $\mathrm{R}$ or $\mathrm{K}$./indicates alternative residues. $\mathrm{X}_{\mathrm{m}-\mathrm{n}}$ indicates any number of unspecified residues from $\mathrm{m}$ to $\mathrm{n}$ in number. (B) Nuclear localization of the full-length proteins PHYSO_357835, PHYSO_480605, PHYSO_251824, PHYSO_561151, and PHYSO_533817 in P. sojae transformants. Representative images. (C) Subcellular localization of PY-NLS-containing segments in P. sojae (left) and in HEK 293 cells (right). (D) HEK 293 cells expressing 2XGFP alone, SV40 NLS-2XGFP, and M9NLS-2XGFP, produced in the same experiment as controls. Representative images are shown in (B-D).

TABLE 1 | Function of PY-NLSs predicted in $P$. sojae nuclear localized proteins.

\begin{tabular}{|c|c|c|c|c|}
\hline FungiDB ID & Annotation & Core PY- NLS-like sequence ${ }^{a}$ & Type $^{b}$ & $\mathrm{Nuc}^{\mathrm{c}}$ \\
\hline PHYSO_357835 & U3 small nucleolar RNA-associated protein & 359 PAPADYTVATTRHKLIIQPY 377 & $\mathrm{~h}$ & $\sqrt{ }$ \\
\hline PHYSO_480605 & Cleavage and polyadenylation specificity factor subunit 3 & 1 MSKRRLAEEAADER $\underline{\mathbf{H} I M R I M P L} 22$ & $\mathrm{~h}, \mathrm{v}$ & $\sqrt{ }$ \\
\hline PHYSO_251824 & $\begin{array}{l}\text { mRNA cleavage and polyadenylation factor I complex, } \\
\text { subunit RNA15 }\end{array}$ & 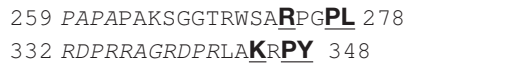 & $\begin{array}{l}h, v \\
b\end{array}$ & $\begin{array}{l}\sqrt{ } \\
\sqrt{ }\end{array}$ \\
\hline PHYSO_561151 & Homeodomain-like transcription factor & 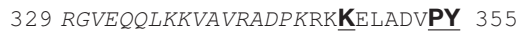 & $\mathrm{b}$ & $x$ \\
\hline PHYSO_533817 & $\mathrm{C} 2 \mathrm{H} 2$ zinc finger protein & 333 RTFKKEDARRQHQLAKHGK $D \underline{\mathbf{P L}} 354$ & $b, v$ & $x$ \\
\hline
\end{tabular}

${ }^{a}$ Epitope 1 that determines the hydrophobic or basic sub-classes of PY-NLSs is highlighted in italics. The R/K/H-X $2-5^{-P Y / L ~ m o t i f ~ i s ~ i n ~ b o l d ~ a n d ~ u n d e r l i n e d . ~}$ ${ }^{b}$ PY-NLS types: b, basic; $h$, hydrophobic; $v$, variant PL epitope.

${ }^{c} \sqrt{ }$ contributes to nuclear localization; $\times$, does not contribute.

To more precisely define the roles of the predicted PY-NLS motifs in the nuclear localization of each of PHYSO_357835, PHYSO_480605, and PHYSO_251824, a series of truncations and mutations were made. These analyses are detailed in the next three sections.

\section{An Augmented PY-NLS Sequence in PHYSO_357835 Is Necessary and Sufficient for Nuclear Import}

The aforementioned experiments indicated that residues 338-387 of PHYSO_357835, containing a predicted PY-NLS sequence, were sufficient to mediate localization of the 2XGFP reporter into the $P$. sojae nucleus. However, examination of the PHYSO_357835 protein sequence using PSORT II revealed the presence of another candidate NLS within this segment, namely a monopartite cNLS-like sequence (370-RHKR-373) overlapping with the epitope 2 of the PY-NLS motif (Figure 4A). To test whether nuclear localization by PHYSO_357835338-387 required the putative cNLS separately from the epitope 2 of the PY-NLS-like sequence, histidine at 371 and lysine at 372 were mutated to alanines, resulting in 370-RAAR-373 (the canonical PY-NLS consensus requires only a single basic residue at this position). Although 2XGFP-PHYSO_357835338-387 


\section{A PHYSO_357835}

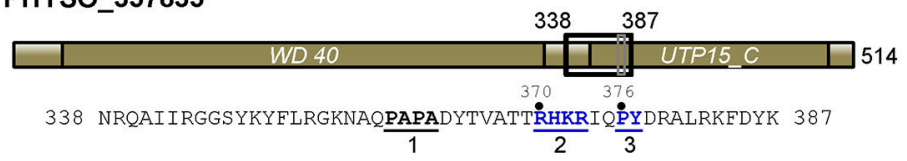

B 2XGFP-PHYSO_357835 $338-387$
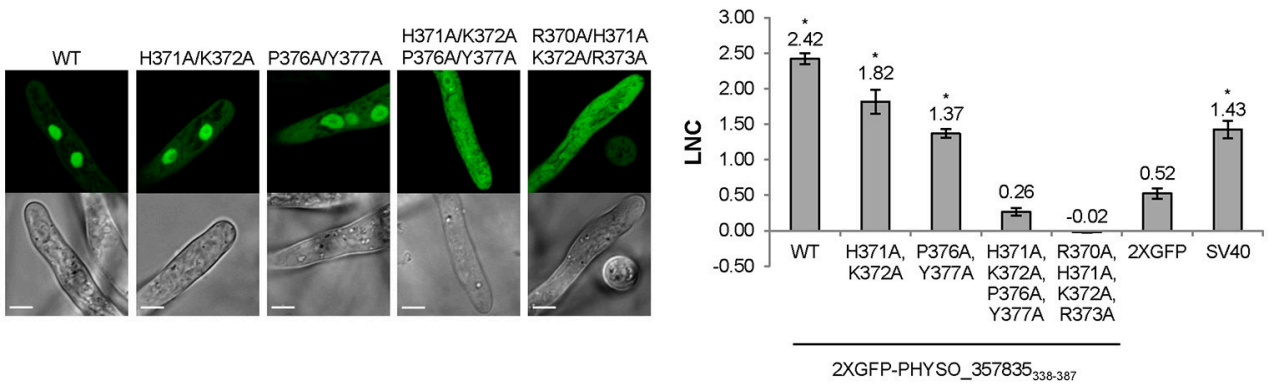

C PHYSO_357835-GFP
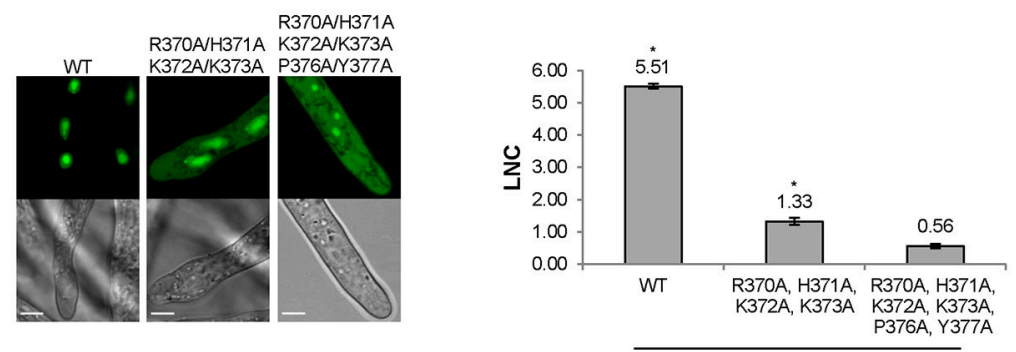

PHYSO_357835-GFP

FIGURE 4 | Nuclear localization of PHYSO_357835 is mediated by a PY-NLS that incorporates a cNLS-like motif. (A) Domain structure of PHYSO_357835. The position of the PY-NLS and the predicted cNLS within PHYSO_357835 are indicated by a black and a gray rectangle, respectively, and the corresponding amino acid sequence is listed below. The three PY-NLS epitopes are in bold, underlined, and numbered. Blue residues indicate the PY motif and basic region corresponding to the predicted cNLS, which were subjected to mutational analysis. No NLS sequences were predicted by NLStradamuS or cNLS Mapper. (B) Subcellular localization of PHYSO_357835 mutants in the context of the C-terminal domain, 338-387. Left, representative images from P. sojae transformants expressing various mutations of 2XGFP-PHYSO_357835338-387. Right, quantification of the localization of 2XGFP-PHYSO_357835338-387 fusion proteins. (C) Subcellular localization of PHYSO_357835 mutants in the context of full length PHYSO_357835-GFP. Left, representative images; right, quantitation. The dots observed in P. sojae hyphae expressing PHYSO_357835-GFP-(R370A/H371A/K372A/R373A/P376A/Y377A) may be nucleoli as the WT shows substantial nucleolar localization, but this was not verified. The LNC for this mutant was calculated assuming the dots were nucleoli. Asterisks, LNC-values of NLS-2XGFP fusions that are significantly greater (FDR $p^{\prime}<$ 0.001 ) than the LNC of 2XGFP.

(H371A/K372A) appeared slightly more cytoplasmic than wild type ( $\mathrm{LNC}=1.82$ compared to 2.42 ), the reporter remained primarily nuclear, suggesting that the nuclear localization of PHYSO_357835338-387 was not primarily dependent on this putative cNLS.

To confirm whether the predicted PY-NLS sequence within PHYSO_357835338-387 was the only NLS that determined the nuclear localization of PHYSO_357835338-387, amino acid substitutions were introduced at the key residues in the predicted PY-NLS sequence. As shown in Figure 4B, mutation of the PY residues (P376A/Y377A) reduced but did not eliminate nuclear localization (LNC $=1.37$ ). However, when the cNLS mutations H371A/K372A were combined with the PY mutations, nuclear localization produced by PHYSO_357835338-387 was abolished ( $\mathrm{LNC}=0.26$; Figure $4 \mathrm{~B}$ ), suggesting that the PY motif, in combination with the cNLS, is required for the nuclear localization of PHYSO_357835338-387. To further explore the role of the basic region (370-RHKR-373), all the four basic residues were converted to alanines. This mutation also abolished nuclear localization ( $\mathrm{LNC}=-0.02$ ), indicating that the full set of four residues of this basic region is essential for nuclear import. Together these results suggest that both the predicted PY-NLS (defined by the residues PY in combination with at least 1 of RHKR) and the predicted cNLS (defined by all four of RHKR) are required for efficient nuclear localization of this $P$. sojae protein.

To test the role of this cNLS-augmented PY-NLS sequence in import of the full length PHYSO_357835 protein, mutations in the basic region (R370A/H371A/K372A/R373A) alone and combination with the $\mathrm{PY}$ dipeptide (R370A/H371A/K372A/R373A/P376A/Y377A) were introduced into full-length GFP-tagged protein. Mutation of the basic region resulted in substantial mislocalization of the full 
length protein into the cytoplasm (from LNC of 5.51 to 1.33), and additional mutation of the PY motif further decreased the LNC-value (to 0.56 , not significantly different than 2XGFP alone; FDR $p^{\prime}=0.7$; Figure 4C). Together, these results indicate that the augmented PY-NLS sequence of PHYSO_357835 is necessary as well as sufficient for the nuclear localization of this protein. Other than PSORT II, which predicted the RHKR element of this NLS, no current software programs, including NLStradamus or cNLS Mapper, predicted the functional NLS sequences of PHYSO_357835 (Tables S3, S4).

\section{Nuclear Import of PHYSO_480605 Is Mediated by a Variant PY-NLS}

As noted above, the predicted PY-NLS located at 1-32 within PHYSO_480605 contains terminal PL residues rather than PY residues. To test if this variant motif is required for PHYSO_480605 nuclear localization, the protein was split at position 32 and each fragment was fused to $2 \mathrm{XGFP}$ and expressed in $P$. sojae. The $\mathrm{N}$-terminal 32 residues fused to $2 \mathrm{XGFP}$ exhibited significant nuclear localization with some visible cytoplasmic signal $\left(\mathrm{LNC}=1.48\right.$, FDR $p^{\prime}<0.001$; Figures 2B, 5B), while the C-terminal fragment (residues 33-754) was exclusively distributed in the cytoplasm (Figure 5B). These results indicated that the fragment containing the variant PY-NLS was necessary for the nuclear import of PHYSO_480605, but suggested that additional amino acids downstream of the motif may contribute to the strength of the nuclear targeting (LNC of the full length protein was 5.79). In fact, an expanded fragment (1-60) that includes a stretch of positively charged amino acids (36-KFKGK40) showed significantly increased nuclear localization (from $\mathrm{LNC}=1.48$ to 2.18 , FDR $p^{\prime}<0.001$; Figure 5B). However, localization was still much less than the full length protein $(\mathrm{LNC}=5.79)$, suggesting that additional downstream sequences might augment nuclear localization, despite being insufficient to independently direct localization. No current software programs, including PSORT II, NLStradamus or cNLS Mapper, predicted any NLS sequences in PHYSO_357835 (Tables S3, S4).

\section{Nuclear Localization of PHYSO_251824 Requires Collaboration of Three Distinct NLS-Like Sequences within the C-terminus} As noted above, residues 239-358 of PHYSO_251824 produced only weak nuclear localization (LNC $=0.90$, FDR $p^{\prime}<$ 0.002; Figures 3C, 6C). To better identify the sequences required for localization, we generated fusions containing larger protein fragments of PHYSO_251824. The entire Nterminal (1-238) and C-terminal (239-419) segments of PHYSO_251824 were expressed as fusions with 2XGFP in P. sojae transformants. PHYSO_251824 $1-238_{-2}$ XGFP showed strictly cytoplasmic distribution (LNC $=-0.52$ ), despite possessing a putative monopartite cNLS (cNLS1, 100-RKRH103) (Figures 6A,B). In contrast, 2XGFP-PHYSO_251824 $239-419$ produced predominant nuclear localization (LNC $=5.30$ compared to 5.38 for the full length protein). Comparison of the localization of 2XGFP-PHYSO_251824 $239-419$ (LNC $=5.30)$ and 2XGFP-PHYSO_251824 $239-358(\mathrm{LNC}=0.90)$ indicated that residues 359-419 must contribute to NLS function (Figure 6C). Examination of those residues using PSORT II revealed another predicted monopartite cNLS (363-PSKRSKP369, cNLS2) (Figure 6A). To test whether cNLS2 was necessary for nuclear localization produced by PHYSO_251824 239-419, the basic amino acids in cNLS2 were all substituted with alanines (K365A/R366A/K368A); this mutation dramatically reduced the

A PHYSO_480605
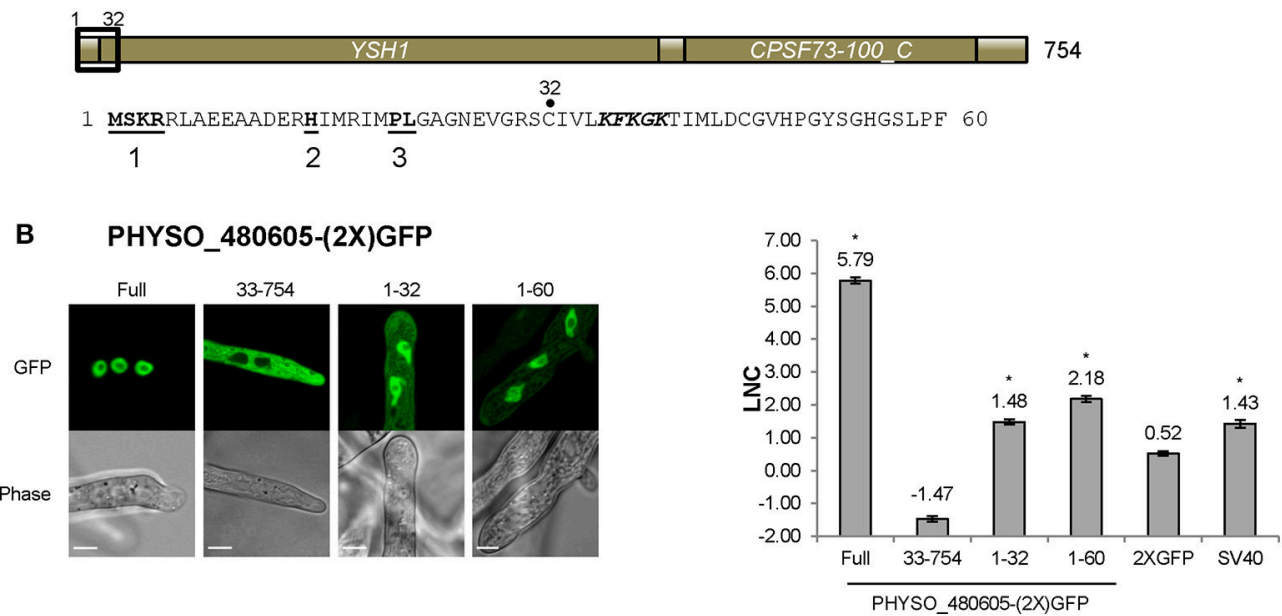

FIGURE 5 | Nuclear localization of PHYSO_480605 requires a region containing a PY-NLS with a variant PY motif. (A) Domain structure of PHYSO_480605. Position of the predicted PY-NLS is indicated by a black rectangle and the corresponding sequence is listed below. The three PY-NLS epitopes are in bold and underlined. The basic patch corresponding to a predicted CNLS is in bold and italics. No NLS sequences were predicted by PSORT II, NLStradamus or cNLS Mapper. (B) Subcellular localization of 2XGFP with full length PHYSO_480605 or fragments of it in P. sojae transformants. Left, representative images; right, quantification. Asterisks, LNC-values of NLS-(2X)GFP fusions that are significantly greater (FDR $\left.p^{\prime}<0.001\right)$ than the LNC 2XGFP. 


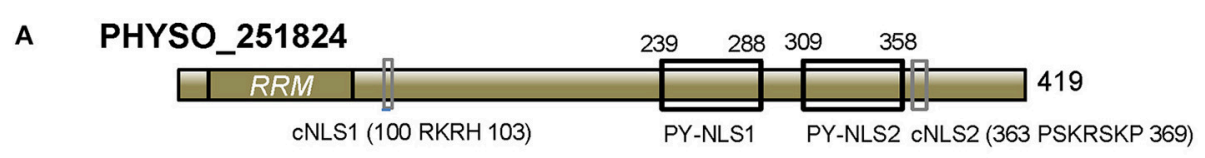

239 GPGILGMAP PGVQPGMMNPAPAPAPAKSGGTRWSARP GPLAAQAT S SAMGGRPAVGI PAS S PMHAQPQPG

309 LMPTPTGPPQSSGPPSSNLAHASRDPRRAGRDPRLAKRPYPGEQGLAPPTGDNDPSKRSKPSGFGADAAP

GGQFDAIAELARDMTPQKLDMLPPNERQMLLAFMQQNNIPF419
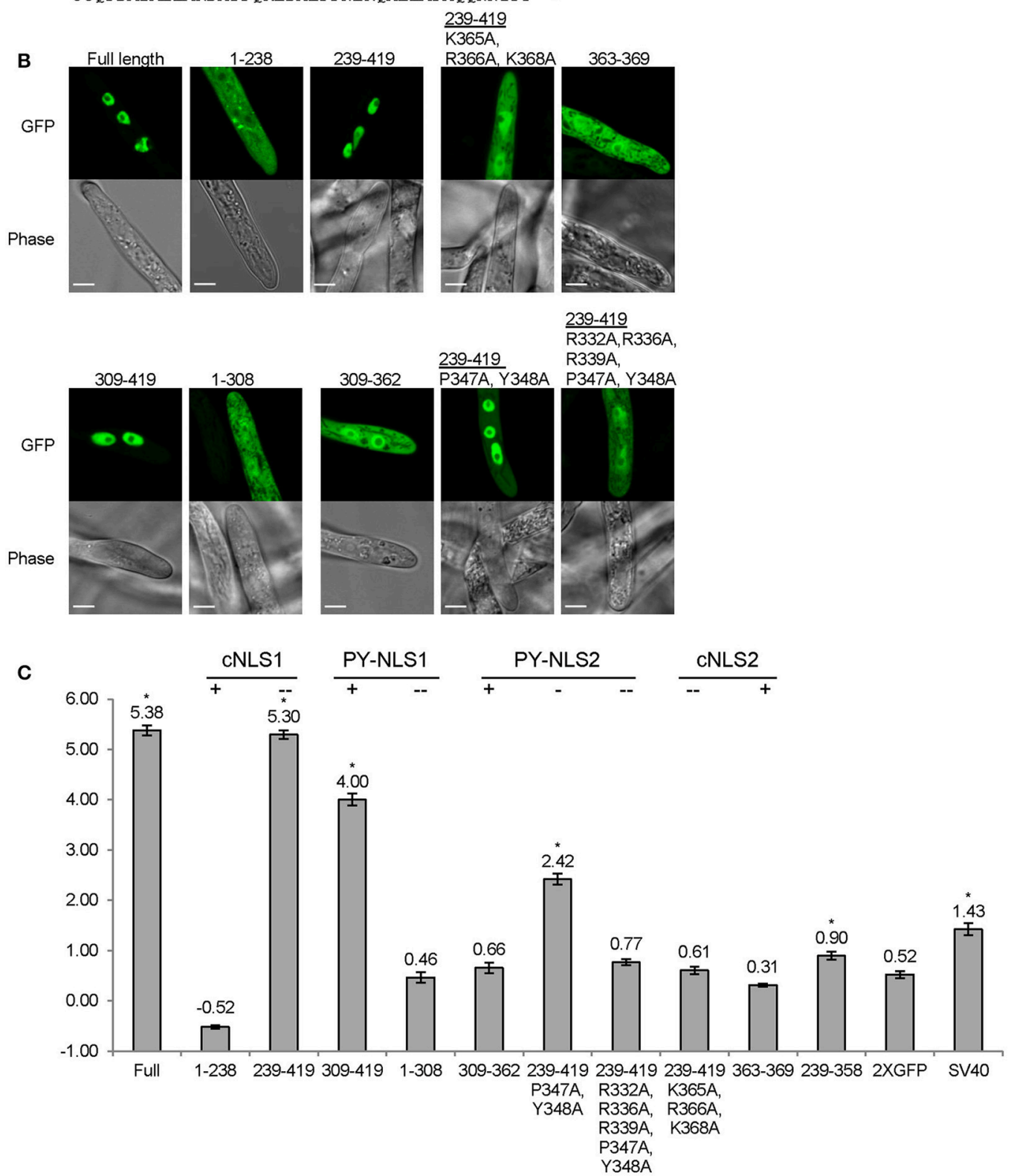

FIGURE 6 | Nuclear localization of PHYSO_251824 requires contributions from two PY-NLS and one cNLS clustered within the C-terminus. (A) Domain structure of PHYSO_251824. The two candidate PY-NLSs and the two PSORT II-predicted cNLSs are indicated by black and gray rectangles respectively; their corresponding sequences are shown below. Predicted cNLS1 is inactive. The three epitopes of the two PY-NLS sequences are in bold and underlined. Amino acids subjected to mutational analysis are in blue. Amino acid sequences underlined by gray solid, dash, and dotted lines indicate the NLSs predicted by PSORT II, NLStradamus, and cNLS Mapper respectively. (B) Subcellular localization of PHYSO_251824-GFP and mutants fused to 2XGFP in P. sojae transformants. N-terminal truncations and cNLS2 were fused to 2XGFP at their C-termini, while various C-terminal PHYSO_251824 truncations were fused to 2XGFP at their N-termini. Representative images are shown. (C) Quantification of localization of PHYSO_251824 mutants. Image of PHYSO_251824239-358 is shown in Figure 3C. Mutational statuses of the various NLS candidates are labeled at the top. +, NLS candidate is the only one in the segment; -, NLS candidate is partially mutated; --, NLS candidate is completely mutated. Asterisks, LNC-values of NLS-2XGFP fusions that are significantly greater (FDR $\left.p^{\prime}<0.001\right)$ than the LNC of 2XGFP. 
nuclear localization of 2XGFP-PHYSO_251824 $239-419$ (LNC = 0.61 vs. 5.30; Figures 6B,C). To test whether cNLS2 was sufficient for nuclear localization, residues 363-369 were fused to 2XGFP; however these residues alone were not sufficient to direct 2XGFP into the nucleus (LNC $=0.31$; Figures 6B,C). Thus, cNLS2 was revealed to be a non-autonomous enhancer of nuclear localization by PHYSO_251824 $239-419$.

In addition to cNLS2, residues 239-419 of PHYSO_251824 contain two predicted PY-NLS motifs. PY-NLS1 is located at positions 239-288 and has a variant PY motif (PL), while PYNLS2 is a conventional PY-NLS located 309-358 (Figure 6A). To test the contribution of PY-NLS1, residues 239-308 were deleted. The resulting segment, 2XGFP-PHYSO_251824 $309-419$ showed a significantly reduced localization compared to 2XGFPPHYSO_251824239-419 $\left(\mathrm{LNC}=4.00\right.$ vs. 5.30, FDR $p^{\prime}<0.001$; Figure 6C). This finding suggested that PY-NLS1 may have weak NLS activity. In support of this conclusion, extension of residues 239-308 onto PHYSO_2518241-238-2XGFP resulted in the reappearance of some nuclear localization (LNC = 0.46 vs. -0.36 , FDR $p^{\prime}<0.001$; Figures 6B,C). To address the contribution of PY-NLS2, specific amino acids were converted into alanines in the PY-NLS2. In the context of residues 239-419, substitution of the PY residues of PY-NLS2 to alanines (P347A/Y348A) partially reduced the nuclear localization (from $\mathrm{LNC}=5.30$ to 2.42 , FDR $p^{\prime}<$ 0.001 ), while additional substitutions in the basic epitope (R332A/R336A/R339A/P347A/Y348A) effectively eliminated the nuclear localization (LNC of 0.77 , not significantly greater than 2XGFP alone; Figures 6B,C). On the other hand, the PY-NLS2 alone (309-362) was not sufficient to direct 2XGFP into the nucleus (LNC $=0.66$; Figures 6B,C), suggesting that it is necessary but not sufficient for efficient nuclear localization.

Taken together, these findings indicate the nuclear localization of PHYSO_251824 is determined by the C-terminal region, in which two weak PY-NLSs and one cNLS-like enhancer sequence operate synergistically to this protein into the nucleus. No current software programs, including PSORT II, NLStradamus or cNLS Mapper, fully predicted the functional NLS sequences of PHYSO_251824 (Tables S3, S4). PSORT II predicted cNLS2, NLStradamus predicted a fragment of PY-NLS2, while cNLS Mapper predicted a fragment of PY-NLS2 in combination with cNLS2.

\section{Highly Conserved Nuclear-localized Proteins Show Different Sequence Requirements for Nuclear Import in P. sojae than in Human and Yeast Counterparts}

To examine whether $P$. sojae utilizes the same nuclear import sequences for transport of conserved nuclear-localized proteins, we examined ribosomal proteins and core histones. Newly synthesized ribosomal proteins are transported into the nucleus in order to assemble with rRNAs in the nucleolus (Lafontaine and Tollervey, 2001; Gerhardy et al., 2014). Histones, including H2A, $\mathrm{H} 2 \mathrm{~B}, \mathrm{H} 3, \mathrm{H} 4$, together with the linker histone $\mathrm{H} 1$, are essential components of chromatin (Baake et al., 2001).
We first examined the $P$. sojae ribosomal protein PsL28 which is the ortholog of the yeast ribosomal protein L28 (ScL28, former name L29, Underwood and Fried, 1990; the naming system for $P$. sojae ribosomal proteins follows the nomenclature of S. cerevisiae; Mager et al., 1997). Protein sequence alignment revealed that the N-termini of the L28 orthologs are highly conserved among different organisms (Figure 7A). Based on functional assays, ScL28 was reported to contain two NLSs: ScL28-NLS1, located at amino acid residues 713, and ScL28-NLS2 at 24-30 (Underwood and Fried, 1990). The sequence corresponding to NLS1 in PsL28 showed two amino acid differences from ScL28-NLS1, while the NLS2 sequence was exactly the same in all L28 orthologs (Figure 7A). The minimal conserved region of PsL2 $8_{1-33}$ that contains both NLSs was expressed as a fusion with 2XGFP and showed strong nuclear localization (Figure 7A). In contrast, when each putative NLS was tested separately by fusion with 2XGFP, neither one produced nuclear localization in $P$. sojae transformants (Figure 7A). However, as indicated above (Figures 1E,F), the two sequences in PsL28 corresponding to NLS1 and NLS2 together constitute an extended bipartite NLS that includes a core bipartite NLS motif (residues 11-27) together with additional flanking positive residues at the N-terminus; this extended bipartite NLS is functional even though the core bipartite NLS [PsL28 $1-33(\mathrm{~K} 7 \mathrm{~A} / \mathrm{R} 9 \mathrm{~A} / \mathrm{K} 10 \mathrm{~A})]$ is not (Figures 1E,F). Both NLStradamus and cNLS mapper correctly predicted this extended bipartite cNLS (Tables S3, S4).

We also tested two other ribosomal proteins, S22a and L3. Segments 21-29 of P. sojae S22a (PsS22a) and 1-22 of P. sojae L3 (PsL3), contain residues that are nearly identical to sequences that produced nuclear localization in yeast (Moreland et al., 1985; Timmers et al., 1999). However, in P. sojae transformants these segments produced only cytoplasmic accumulation (Figure S4); addition of flanking sequences (PsS22 $\mathrm{a}_{1-34}$ and $\mathrm{PsL}_{1-36}$ ) had no effect on nuclear localization either. Thus, other or additional sequences appear to be required for nuclear localization of these proteins in P. sojae. PSORT II and NLStradamus incorrectly predicted PsL3 ${ }_{1-36}$ should be nuclear localized, but $c N L S$ Mapper did not. All three predicted that PsS22 $\mathrm{a}_{1-34}$ would not be nuclear localized and PSORT II predicted the presence of a cNLS motif elsewhere in the protein (117-RRKH-120) (Table S4).

A similar strategy was carried out to determine the activities of putative NLSs in core histones $\mathrm{H} 3$ and $\mathrm{H} 4$, which are nearly identical in amino acid sequences in all eukaryotes (Figures 7B,C). The NLSs in $\mathrm{H3}$ and $\mathrm{H} 4$ have been experimentally characterized in human and yeast (Figures 7B,C). There is only one NLS reported in each of yeast $\mathrm{H} 3(\mathrm{ScH} 3)$ and H4 (ScH4) (Mosammaparast et al., 2002), while more than one NLS has been found in their human counterparts (Baake et al., 2001). The NLSs in ScH3 and ScH4 somewhat overlapped with their human orthologs, suggesting a common origin.

A series of truncations were made of $P$. sojae $\mathrm{H} 3$ (PsH3) and $\mathrm{H} 4$ ( $\mathrm{PsH} 4$ ) according to the NLS locations in the yeast and human orthologs. The resulting fragments were tested for nuclear localization as 2XGFP fusions. As expected, the fulllength $\mathrm{PsH} 3$ and $\mathrm{PsH} 4$ reporter constructs produced strong 
A

AtL27a-3
HsL27a
ScL28
PsL28

MTTRFKKNRKKRGHVSAGHGRIGKHRKHPGGRG MPSRLRKTRKLRGHVSHGHGRIGKHRKHPGGRG MPSRFTKTRKHRGHVSAGKGRIGKHRKHPGGRG MPSRFSKNRKKRGHVSAGHGRIGKHRKHPGGRG $\begin{array}{rrr}1 & 7113 & 24273033\end{array}$
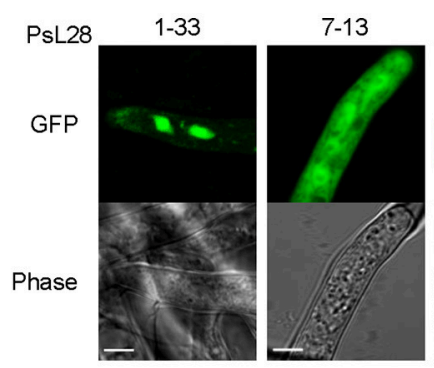

24-30

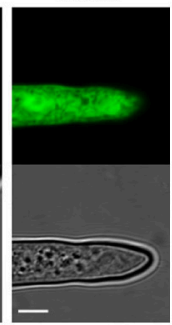

B AtH3.1

$\mathrm{HsH} 3 . \mathrm{k}$

$\mathrm{ScH} 3 / \mathrm{Hht} 2 \mathrm{p}$

$\mathrm{PsH} 3$

MARTKQTARKSTGGKAPRKQLATKAARKSAPATGGVKKPHRFRPGTVALRE IRKYQKSTELLIRKLPFQRLVREI MARTKQTARKSTGGKAPRKQLATKAARKSAPATGGVKKPHRYRPGTVALREIRRYQKSTELLIRKLPFQRLVREI MARTKQTARKSTGGKAPRKQLASKAARKSAPSTGGVKKPHRYKPGTVALRE IRRFQKSTELLIRKLPFQRLVREI MARTKQTARKSTGGKAPRKQLATKAARKSAPATGGVKKPHRYRPGTVALRE IRRYQKSTE LLIRKLPFQRLVREI 1 2832 75

AtH3. 1

$\mathrm{HsH} 3 . \mathrm{k}$

$\mathrm{ScH} 3 / \mathrm{Hht} 2 \mathrm{p}$

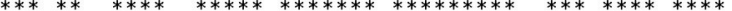

AQDFKTDLRFQSSAVAALQEAAEAYLVGLFEDTNLCAIHAKRVTIMPKDIQLARRIRGERA AQDFKTDLRFQSSAVMALQEACEAYLVGLFEDTNLCAIHAKRVTIMPKDIQLARRIRGERA AQDFKTDLRFOSSAIGALOESVEAYLVSLFEDTNLAAIHAKRVTIOKKDIKLARRLRGERS AQDFKTDLRFQGSAVLALQEAAEAYLVGLFEDTNLCAIHAKRVTIMPKDIQLARRIRGERS

$\mathrm{PSH} 3$ 116 136

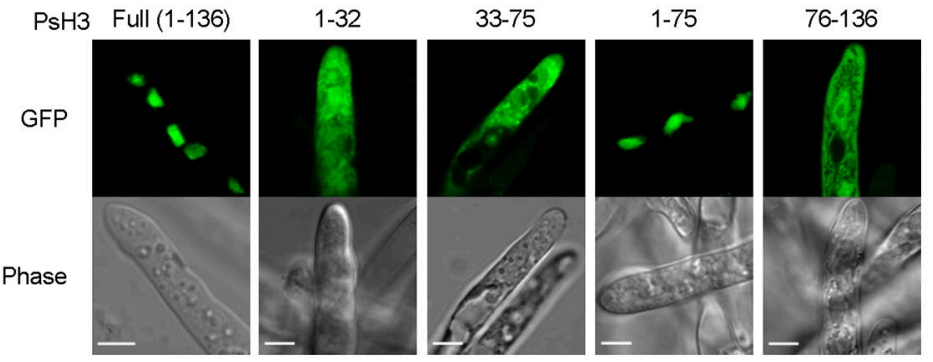

C

\section{AtH4 \\ $\mathrm{HsH} 4 . \mathrm{j}$ $\mathrm{ScH} 4 / \mathrm{Hhf} 2 \mathrm{p}$ \\ $\mathrm{Ps} 44$}

AtH4

$\mathrm{HsH} 4 . \mathrm{j}$

$\mathrm{ScH} 4 / \mathrm{Hhf} 2 \mathrm{p}$

$\mathrm{P} 5 \mathrm{H} 4$

MSGRGKGGKGLGKGGAKRHRKVLRDNIQGITKPAIRRLARRGGVKRISGLIYEETRGVL MSGRGKGGKGLGKGGAKRHRKVLRDNIQGITKPAIRRLARRGGVKRISGLIYEETRGVLK MSGRGKGGKGLGKGGAKRHRKI LRDNIQGITKPAIRRLARRGGVKRISGLIYEEVRAVLK MSGRGKGGKGLGKGGAKRHRKVLRDNIQGITKPAIRRLARRGGVKRISGLIYEETRGVLK 1

IFLENVIRDAVTYTEHARRKTVTAMDVVYALKRQGRTIYGFGG VFLENVIRDAVTYTEHAKRKTVTAMDVVYALKRQGRTLYGFGG SFLESVIRDSVTYTEHAKRKTVTSLDVVYALKRQGRTLYGFGG VFLENVIRDSVTYTEHARRKTVTAMDVVYALKRQGRTLYGFGG
80

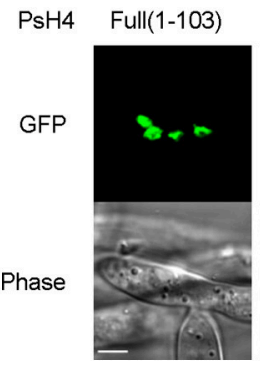

$1-25$

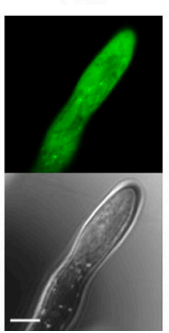

$1-42$

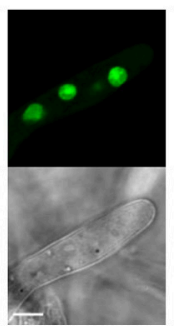

$1-80$

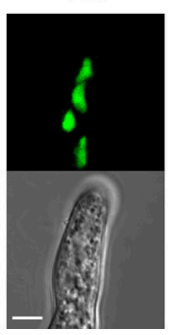

26-42

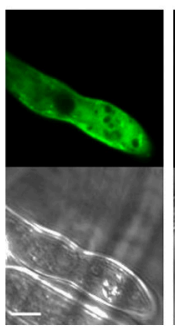

26-80

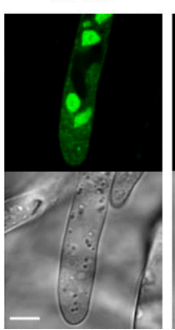

43-103

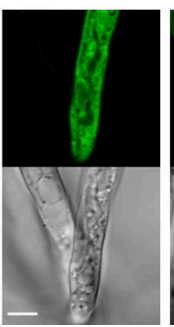

81-103

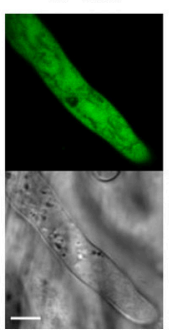

FIGURE 7 | Combinatorial usage of NLSs for nuclear transport of $\boldsymbol{P}$. sojae ribosomal protein L28 and core histones H3 and H4. (A-C) Upper panels, alignments of P. sojae ribosomal proteins L28 (PsL28, PHYSO_355737) and core histones H3 (PsH3, PHYSO_286415) and H4 (PsH4, PHYSO_285922) with their Arabidopsis thaliana (At), human (Hs), and Saccharomyces cerevisiae (Sc) orthologs. Asterisks on the top of each alignment indicate conserved residues among L28, $\mathrm{H} 3$, or $\mathrm{H} 4$ orthologs. Regions individually conferring nuclear targeting in human core histones are highlighted in green, cyan, or gray while those required in yeast are highlighted in yellow. NLSs predicted in the P. sojae core histone and ribosomal protein by PSORT II, NLStradamus, and NLS Mapper are underlined by gray solid, dash, and dotted lines respectively. Lower panels, subcellular localization of various PsL28, PsH3, or PsH4 truncations. Representative images are shown. 
nuclear localization (Figures 7B,C). However, none of the $\mathrm{H} 3$ fragments responsible for nuclear localization in yeast or human (residues 1-32 or residues 33-75) individually exhibited the same NLS activities in $P$. sojae transformants. Region 1-75 of PsH3, which encompasses both of the two NLS regions of human $\mathrm{H} 3$, did produce strong nuclear localization in $P$. sojae (Figure 7B). Expression of a $P$. sojae $\mathrm{H} 3$ segment that lacked the $\mathrm{N}$-terminal domain, $\mathrm{H} 3_{76-136}$, did not produce nuclear localization (Figure 7B), indicating that no other sequences contribute to $\mathrm{PsH} 3$ nuclear localization. NLStradamus predicted part of this region (10-36) but not all of it. PSORT II and cNLS mapper did not predict this region (Tables S3, S4).

In the case of $\mathrm{PsH} 4$, residues 1-25, which produced partial NLS activity in yeast (Mosammaparast et al., 2002) and full activity in human cells (Baake et al., 2001), produced no observable nuclear localization in $P$. sojae (Figure 7C). A larger N-terminal GFP fusion $\mathrm{PsH}_{1-42}$-2XGFP produced clear nuclear localization in $P$. sojae with little detectable fluorescence in the cytoplasm, while residues $1-80$ of $\mathrm{PsH} 4$ produced complete nuclear localization (Figure 7C). This result suggests that segment 26-42 of PsH4, which was identified as an NLS in human cells, contains determinants that may contribute to NLS function in $P$. sojae. However, $\mathrm{PsH}_{26-42}$ alone was insufficient to mediate nuclear localization of the GFP reporter, indicating that these residues could not act as an independent NLS (Figure 7C). On the other hand, $\mathrm{PsH}_{26-80}$, which spans two regions (2642 and 43-80) with NLS activity in human, did produced some nuclear localization in $P$. sojae but less than 1-80 (Figure 7C), whereas 43-103 showed no activity (Figure 7C). Together, these results suggest that all three regions corresponding to NLSs in human $\mathrm{H} 4$, namely $1-25,26-42$, and 43-80, must work together in $P$. sojae to produce efficient nuclear localization. $P$. sojae $\mathrm{H} 4$ reporters that lacked the $\mathrm{N}$-terminal domains (H4 $\left.4_{81-103}\right)$ produced no nuclear accumulation (Figure 7C). NLStradamus predicted the first two of these regions, but not the third. PSORT II and cNLS Mapper predicted none of them (Tables S3, S4).

Taken together, the results above suggest that compared to their yeast or human counterparts, $P$. sojae has a similar but stronger set of sequence requirements for translocation of conserved nuclear proteins into the nucleus.

\section{DISCUSSION}

The diverse sequences and structures of NLSs have limited the reliable prediction of nuclear-localized proteins based on amino acid sequence alone. Though consensus sequences have been proposed for some NLS types, such as cNLS and PY-NLS, their application remains constrained by many factors, such as protein context and flanking sequences, and by nuclear import regulation by mechanisms such as phosphorylation and protein interactions (Garcia-Bustos et al., 1991). Moreover, the karyopherins that bind NLSs show differences in specificity from species to species (Marfori et al., 2011), limiting the reliability of NLS consensus sequences when applied to widely divergent species. In this study, after eliminating artifacts caused by staining with DAPI, we identified key differences in several classes of NLSs between the oomycete $P$. sojae on the one hand, and human and yeast on the other hand.

\section{P. sojae Classical NLSs Exhibit Distinct Differences from Human and Yeast}

The cNLSs are the best-characterized class of NLSs to date (Lange et al., 2007). The prototypical monopartite cNLS derived from the SV40 large T antigen (SV40 NLS) has been reported to direct efficient nuclear import in various organisms, including yeast, mammalian cells (Lange et al., 2007), and plants (Chang et al., 2012). However, we found that monopartite cNLSs, represented by the SV40 NLS and by the one from the $c-M y c$ protein, produced relatively weak nuclear localization in $P$. sojae. It has been reported that protein context may influence the activity of NLSs, and multiple copies of a NLS can produce faster, more pronounced nuclear localization (Garcia-Bustos et al., 1991). We showed that SV40 NLS fusions at the C-terminus of GFP, and multiple copies of the NLS increased nuclear accumulation compared to a single copy at the N-terminus of GFP (Figure 1D), although some fluorescence was still visible in the cytoplasm (Figure 1B).

Based on assessment of several putative cNLSs predicted from the consensus sequences in $P$. sojae nuclear proteins, we found that cNLS-mediated nuclear import is conserved in $P$. sojae but that it exhibits some consistent differences: (1) most predicted monopartite cNLSs are not sufficient by themselves for efficient nuclear accumulation of GFP reporters. However, they may function to augment the activity of other NLSs or work collectively with other weak NLSs to accomplish efficient nuclear import of proteins. For example, the monopartite cNLS embedded inside the PY-NLS of PHYSO_357835 was essential for efficient nuclear targeting (Figure 4), while in PHYSO_251824 a C-terminal monopartite cNLS cooperated with two PY-NLSs to direct efficient nuclear localization (Figure 6). (2) With regard to bipartite cNLSs, sequence comparisons and mutational analyses of several functional bipartite cNLSs, including nucleoplasmin, PsL28, and PHYSO_561151, revealed that a functional bipartite cNLS in $P$. sojae typically requires additional basic amino acids in the first sub-motif, compared to the human/yeast consensus and possibly in the second also (Figures 1E,G). We did not test the contributions of non-positively charged residues in the neighborhood of the two positively charged clusters, but there is evidence that flanking residues also may be important for NLS activity (Kosugi et al., 2009; Lange et al., 2010).

\section{Efficient PY-NLS-Mediated Nuclear Import Requires Additional Clusters of Basic Amino Acids}

None of the human and yeast PY-NLSs we tested were capable of mediating efficient nuclear entry of GFP reporters in $P$. sojae, though weak NLS activity was observed with the hnRNP M PY-NLS and the chimeric M9M peptide. Given that the M9M peptide is a hPY-NLS (Figure 2A), our findings suggest that, in contrast to yeast, the $P$. sojae nuclear transport machinery may have evolved to transport both bPY-NLS and hPY-NLS cargos. 
To characterize $P$. sojae PY-NLSs, we analyzed five native $P$. sojae nuclear-localized proteins which harbored predicted PY-NLS-like motifs. Using deletion and substitution mutations, we identified PY-NLS-containing fragments from three of the proteins that could mediate different degrees of GFP translocation into the nuclei of both $P$. sojae and mammalian cells. In each case however, additional basic amino acids were required, either in association with one of the three PY-NLS epitopes (as in PHYSO_357835), or Cterminal to the PY-NLS motif (PHYSO_480605 and PHYSO_ 251824).

In yeast, Süel et al. (2008) proposed the "degeneracy rule" for the bPY-NLS in which PL is exchangeable with PY. However, yeast Kap $\beta 2$ only recognizes the basic class of PY-NLSs, so PY-motif degeneracy remains unclear for the hydrophobic class of PY-NLSs. Only a few PY-NLSs are reported to have a variant PY motif, such as the RNA-binding protein HuR which contains a PG motif instead of PY. In addition, several newly identified Kap $\beta 2 / K a p 104$ cargos were reported to lack a typical PY motif or even a recognizable PY-NLS (reviewed in Soniat and Chook, 2015). In our study, we found that the variant "PL"-NLS in PHYSO_480605 produced substantial nuclear localization in both $P$. sojae and mammalian cells, suggesting the degeneracy rule may also exist for hPY-NLS.

The PY-NLS "consensus sequence" is defined by a collection of three modular epitopes: an N-terminal hydrophobic or basic residue-enriched motif, a second $\mathrm{R} / \mathrm{K} / \mathrm{H}$ motif and the third P[Y/L] motif (Lee et al., 2006; Süel et al., 2008). Biochemical and biophysical analyses have shown that in different NLSs each epitope may contribute differently to Kap $\beta 2$ (or Kap104p) binding (Lee et al., 2006; Cansizoglu et al., 2007; Süel et al., 2008). For instance, in hnRNP $M$ and in the yeast mRNA processing protein Hrp1, epitope 3 contributes significantly more than the other two epitopes (Cansizoglu et al., 2007; Süel et al., 2008), In contrast, in the M9NLS and Nab2p PY-NLS, epitope 3 contributes weakly to Kap $\beta 2$ (or Kap104p) binding, while strong binding is conferred by epitope 1 or by multiple positions distributed across the three epitopes (Lee et al., 2006; Süel et al., 2008). In our study, we found that mutation of PY (epitope 3) in the PHYSO_357835 PY-NLS sequence reduced but did not abolish nuclear entry, whereas complete abrogation of nuclear entry was observed upon mutation of the basic cluster overlapping epitope 2 . In the case of the PY-NLSs in PHYSO_480605 and PHYSO_251824, an extra basic cluster downstream of the PY-NLS consensus markedly enhanced the nuclear entry, effectively constituting a fourth epitope (Figures 5, 6). These results suggest that extra positive charges may facilitate the binding of PY-NLSs to the presumptive P. sojae Kap $\beta 2$. Similar combinatorial organization of a PY-NLS was observed in the Xenopus Kap $\beta 2$ substrate ELYS (RRTRRRIIAKPVTRRKMR), in which a variant PY dipeptide (PV) is flanked by a Cterminal basic cluster (Lau et al., 2009). Thus, our results may explain why the four PY-NLS prototypes from human and yeast functioned poorly in $P$. sojae, as they lack the additional basic residues required for efficient targeting to the $P$. sojae nucleus.

\section{For Nuclear Import of Highly Conserved Ribosomal and Histone Proteins, P. sojae Requires Combinations of NLSs That Are Autonomous in Other Eukaryotes}

Like the nuclear entry mediated by the P. sojae cNLS and PYNLS motifs, entry by highly conserved nuclear proteins also showed distinctive features in P. sojae. Amino acid sequences of many ribosomal proteins and core histones are highly conserved among different eukaryotes, though within an organism different conserved proteins are imported into the nucleus via different NLSs. This suggests that the responsible karyopherins from different organisms should show similar specificity. However, in $P$. sojae, NLS motifs that could act autonomously in human and yeast were required to act together to deliver conserved proteins into the nucleus. For example, in yeast L28, two sequences (7KTRKHRG-13 and 24-KHRKHPG-30) could individually serve as an NLS. However, the corresponding sequences in P. sojae L28 were required together for efficient nuclear localization (thus constituting a bipartite NLS). In the case of histone $\mathrm{H} 3$, where the amino acid sequences are nearly identical in all eukaryotes, two adjacent sequences that could each serve as an NLS in human (residues 1-32 and 33-75 of H3) were jointly required for NLS activity in $P$. sojae. Similarly, in $\mathrm{H} 4$ three adjacent sequences that could serve separately as NLSs in human were required together in $P$. sojae $\mathrm{H} 4$ for nuclear entry. The results suggest that the $P$. sojae karyopherins that presumably bind to these NLSs do so more weakly than in other characterized eukaryotes, and perhaps therefore that binding by multiple karyopherins is required to produce nuclear entry in $P$. sojae.

\section{Software for Prediction of Functional NLS Regions in Oomycete Proteins}

During the course of this study, we evaluated three software tools for predicting regions of the proteins that might have NLS activity, conferring nuclear localization. PSORT II (PSORT, 1997) was released in 1997, and since then, a variety of experimentally defined NLSs have been found that do not match any of the consensus sequences (Kosugi et al., 2009). Based on those findings, additional NLS prediction tools have been developed to include those newly identified NLS. We evaluated NLStradamus and $c N L S$ Mapper. We also evaluated PredictNLS, but it produced so few predictions as to not be useful, as has been reported elsewhere (Nguyen Ba et al., 2009). Overall we found that NLStradamus and cNLS Mapper identified long regions but with little indication of which amino acid residues might be most responsible for nuclear localization (Tables S3, S4). On the other hand, PSORT II identified large numbers of very specific predicted NLSs; this was useful at the outset of our study when we were searching for sequences important for nuclear localization in $P$. sojae, though in the end many of these proved to be false positives (in P. sojae). After we had experimentally tested a large number of protein segments for nuclear localization activity in P. sojae, it emerged that NLStradamus was most successful in identifying regions that could confer partial or full nuclear localization in P. sojae, albeit only 60\% successful (Tables S3, S4). Of 10 regions predicted in $9 P$. sojae proteins, six were wholly 
or partially correct, while four were incorrect. Of the four, two were false positives and two were false negatives. More accurate prediction tools for oomycetes will need to take into account the combinatorial nature of NLSs we observed in P. sojae, as exemplified most strikingly by PHYSO_251824.

In summary, using $P$. sojae as a model, we identified distinctive features of nuclear localization in oomycetes, based on characterization of multiple classes of NLS from 10 nuclear proteins. A consistent pattern has emerged in which individual NLS sequences that are sufficient for autonomous nuclear localization in other eukaryotes function weakly or not at all in P. sojae, but can collaborate with each other or with patches rich in basic residues to produce efficient localization. Several studies have shown that karyopherins from different families and organisms exhibit preferences for specific NLSs (Sekimoto et al., 1997; Köhler et al., 1999; Fang et al., 2001; Mason et al., 2002; Kosugi et al., 2009; Chang et al., 2012); further characterization of $P$. sojae karyopherin/NLS structures may help us to explain the distinctive features that we observed in our studies. Because nuclear localization has not been dissected comprehensively in a wide diversity of eukaryotes or even in other oomycetes, it is currently unclear whether the differences we observe in $P$. sojae are part of a wider pattern of diversity in eukaryotes, or have a narrower functional significance, related perhaps to the pathogenic lifestyle of this organism.

\section{AUTHOR CONTRIBUTIONS}

YF and BT conceived the study. YF performed $90 \%$ of the experiments. HJ and GW performed human cell transfections; DW contributed technical assistance; YF wrote the manuscript with help of BT. GW also helped to edit the manuscript. All authors reviewed the manuscript and are accountable for its accuracy.

\section{ACKNOWLEDGMENTS}

We thank Anne-Marie Girard (Center for Genome Research and Biocomputing, Oregon State University) for assistance and training with confocal microscopy, Felipe Arredondo (Oregon State University) for assistance and training with $P$. sojae transformation, Danyu Shen (Nanjing Agriculture University) and Tian Hong (University of California, Irvine) for assistance with bioinformatics, and Howard Judelson (University of California, Riverside) for plasmids. We also thank Virginia Tech's Open Access Subvention Fund (to YF) for support of the publication. This work was supported in part by USDA NIFA grant \#2011-68004-30104 to BT.

\section{SUPPLEMENTARY MATERIAL}

The Supplementary Material for this article can be found online at: http://journal.frontiersin.org/article/10.3389/fmicb. 2017.00010/full\#supplementary-material

Figure S1 | Artifacts caused by DAPI (4', 6-diamidino-2-phenylindole) staining for live-cell imaging of $\boldsymbol{P}$. sojae hyphae. To label the $P$. sojae nuclei,
P. sojae transformants expressing candidate NLSs fused to 2XGFP were stained with DAPI (final concentration, $0.2 \mu \mathrm{g} / \mathrm{ml}$ ) for $25 \mathrm{~min}$ before imaging. (A) Different subcellular localizations of the NLS-tagged GFPs in P. sojae transformants with and without DAPI staining. Arrows indicate residual GFP staining of nuclei after DAPI treatment. (B) A representative image showing various localization patterns of 2XGFP fused to a synthetic NLS, PsNLS (Fang and Tyler, 2016). Region (a): GFP reporter showing nuclear localization in a small region of DAPI-stained hyphae. Region (b): Cytoplasmic localization in a larger region of DAPI-stained hyphae; arrowheads indicate artifactual cytoplasmic localization of NLS-GFP fusions. Region (c): Exclusive nuclear accumulation typically found in DAPI-free hyphae. (C) Time-lapse experiment tracking the process of mis-localization of PSNLS-2XGFP upon DAPI treatment. Red rectangles highlight changes occurring during DAPI incubation. In this example, the nuclear-localized PSNLS-2XGFP was released into the cytoplasm following 18-18.5 min incubation with DAPI. Nuclear disintegration occurs at different rates in different regions of the hyphae possibly because newer hyphal regions absorb DAPI more slowly. Also see Supplemental Video 1.

Figure S2 | Detailed mutational analysis of the PY-NLS candidate PHYSO_561151 reveals that an extended bipartite cNLS at the C-terminus is actually responsible for its nuclear localization. (A) Domain structure of PHYSO_561151. Position of the candidate PY-NLS sequence (non-functional) within PHYSO_561151 is indicated by a black rectangle; the shortest bipartite cNLS responsible for the nuclear import of the protein is marked by a gray rectangle. The corresponding amino acid sequences are listed below. Epitopes 1, 2, 3 of the putative PHYSO_561151 PY-NLS sequence are in bold and underlined. NLSs predicted by PSORT II, NLStradamus, and NLS Mapper are underlined by gray solid, dash, and dotted lines respectively. (B) Subcellular localization of PHYSO_561151 and mutants. Representative images are shown.

Figure S3 | Detailed mutational analysis of the PY-NLS candidate PHYSO_533817 reveals that residues 172-314 determine the nuclear accumulation. (A) Domain structure of PHYSO_533817. Position of the candidate PY-NLS (non-functional) sequence within PHYSO_533817 is indicated by a black rectangle. The corresponding amino acid sequence is listed below. Epitopes 1, 2, 3 of the putative PY-NLS sequence are in bold and underlined. NLSs predicted by PSORT II, NLStradamus, and NLS Mapper are indicated by gray solid and dash lines respectively. (B) Subcellular localization of PHYSO_533817 and mutants. Representative images are shown.

Figure S4 | Sequences used for nuclear import of ribosomal proteins S22a and L3 in yeast do not show the same activities in $P$. sojae. (A,B) Left panels, alignment of $P$. sojae ribosomal proteins S22a (PsS22a, PHYSO_287103) and L3 (PsL3, PHYSO_285779), with their orthologs in Arabidopsis thaliana (At), human (Hs), and Saccharomyces cerevisiae (Sc), respectively. Asterisks on the top of each alignment indicate conserved residues among the S22a or L3 orthologs. Sequence highlighted in yellow, NLSs reported in yeast ribosomal proteins S22a (Timmers et al., 1999) and L3 (Moreland et al., 1985). No NLS sequences were predicted by PSORT I/, NLStradamus or cNLS Mapper in PsS22a. In PsL3, NLSs predicted by PSORT /| and NLStradamus are underlined by gray solid, dash lines respectively; no NLS sequences were predicted by cNLS Mapper. Right panels, various fragments of ribosomal proteins, were expressed as fusions to 2XGFP in $P$. sojae transformants and visualized by confocal microscopy. Representative images are shown.

Supplemental Video 1 | Mis-localization of PsNLS-2XGFP occurred in $P$. sojae hyphae during DAPI treatment. Red rectangle highlights changes occurring during DAPI incubation.

Data Sheet 1 | Supplemental sequences of the five putative PY-NLS-containing proteins.

Table S1 | Phytophthora protein identifiers in FungiDB and NCBI.

Table S2 | Primers used in this study.

Table S3 | Summary of conventional and predicted cNLS-containing fragments that were tested throughout this study.

Table S4 | NLS predicted by different NLS predictors. 


\section{REFERENCES}

Ah-Fong, A. M., and Judelson, H. S. (2011). Vectors for fluorescent protein tagging in Phytophthora: tools for functional genomics and cell biology. Fungal Biol. 115, 882-890. doi: 10.1016/j.funbio.2011. 07.001

Baake, M., Bauerle, M., Doenecke, D., and Albig, W. (2001). Core histones and linker histones are imported into the nucleus by different pathways. Eur. J. Cell Biol. 80, 669-677. doi: 10.1078/0171-9335-00208

Benjamini, Y., and Hochberg, Y. (1995). Controlling the false discovery rate - a practical and powerful approach to multiple testing. J. R. Statist. Soc. Ser. B Methodol. 57, 289-300.

Bonifaci, N., Moroianu, J., Radu, A., and Blobel, G. (1997). Karyopherin $\beta 2$ mediates nuclear import of a mRNA binding protein. Proc. Natl. Acad. Sci. U.S.A. 94, 5055-5060. doi: 10.1073/pnas.94.10.5055

Cansizoglu, A. E., Lee, B. J., Zhang, Z. C., Fontoura, B. M., and Chook, Y. M. (2007). Structure-based design of a pathway-specific nuclear import inhibitor. Nat. Struct. Mol. Biol. 14, 452-454. doi: 10.1038/nsmb1229

Chang, C. W., Counago, R. L., Williams, S. J., Boden, M., and Kobe, B. (2012). Crystal structure of rice importin- $\alpha$ and structural basis of its interaction with plant-specific nuclear localization signals. Plant Cell 24, 5074-5088. doi: $10.1105 /$ tpc.112.104422

Chook, Y. M., and Suel, K. E. (2011). Nuclear import by karyopherinBs: recognition and inhibition. Biochim. Biophys. Acta 1813, 1593-1606. doi: 10.1016/j.bbamcr.2010.10.014

Cokol, M., Nair, R., and Rost, B. (2000). Finding nuclear localization signals. EMBO Rep. 1, 411-415. doi: 10.1093/embo-reports/kvd092

Dingwall, C., and Laskey, R. A. (1991). Nuclear targeting sequences-a consensus? Trends Biochem. Sci. 16, 478-481. doi: 10.1016/0968-0004(91)90184-W

Dingwall, C., Sharnick, S. V., and Laskey, R. A. (1982). A polypeptide domain that specifies migration of nucleoplasmin into the nucleus. Cell 30, 449-458. doi: 10.1016/0092-8674(82)90242-2

Erwin, D. C., and Ribeiro, O. K. (1996). Phytophthora Diseases Worldwide. American Phytopathological Society. St. Paul, MN: APS Press.

Fang, X., Chen, T., Tran, K., and Parker, C. S. (2001). Developmental regulation of the heat shock response by nuclear transport factor karyopherin- $\alpha 3$. Development 128, 3349-3358.

Fang, Y., and Tyler, B. M. (2016). Efficient disruption and replacement of an effector gene in the oomycete Phytophthora sojae using CRISPR/Cas9. Mol. Plant Pathol. 17, 127-139. doi: 10.1111/mpp.12318

Gamboa-Meléndez, H., Huerta, A. I., and Judelson, H. S. (2013). bZIP transcription factors in the oomycete Phytophthora infestans with novel DNAbinding domains are involved in defense against oxidative stress. Eukaryotic Cell 12, 1403-1412. doi: 10.1128/EC.00141-13

Garcia-Bustos, J., Heitman, J., and Hall, M. N. (1991). Nuclear protein localization. Biochim. Biophys. Acta 1071, 83-101. doi: 10.1016/0304-4157(91) 90013-M

Gerhardy, S., Menet, A. M., Peña, C., Petkowski, J. J., and Panse, V. G. (2014). Assembly and nuclear export of pre-ribosomal particles in budding yeast. Chromosoma 123, 327-344. doi: 10.1007/s00412-014-0463-z

Hardham, A. R. (2001). "Investigations of oomycete cell biology," in Molecular and Cellular Biology of Filamentous Fungi: A Practical Approach, ed N. J. Talbot (New York, NY: Oxford University Press), 127-155.

Horton, P., Park, K. J., Obayashi, T., Fujita, N., Harada, H., Adams-Collier, C. J., et al. (2007). WoLF PSORT: protein localization predictor. Nucleic Acids Res. 35(Web Server issue), W585-W587. doi: 10.1093/nar/gkm259

Hunter, C. C., Siebert, K. S., Downes, D. J., Wong, K. H., Kreutzberger, S. D., Fraser, J. A., et al. (2014). Multiple nuclear localization signals mediate nuclear localization of the GATA transcription factor AreA. Eukaryot Cell 13, 527-538. doi: 10.1128/EC.00040-14

Judelson, H. S., and Blanco, F. A. (2005). The spores of Phytophthora: weapons of the plant destroyer. Nat. Rev. Microbiol. 3, 47-58. doi: 10.1038/nrmicro1064

Kalderon, D., Richardson, W. D., Markham, A. F., and Smith, A. E. (1983). Sequence requirements for nuclear location of simian virus 40 large-T antigen. Nature 311, 33-38. doi: 10.1038/311033a0

Kalderon, D., Roberts, B. L., Richardson, W. D., and Smith, A. E. (1984). A short amino acid sequence able to specify nuclear location. Cell 39, 499-509. doi: 10.1016/0092-8674(84)90457-4
Kamoun, S., Furzer, O., Jones, J. D., Judelson, H. S., Ali, G. S., Dalio, R. J., et al. (2015). The Top 10 oomycete pathogens in molecular plant pathology. Mol. Plant Pathol. 16, 413-434. doi: 10.1111/mpp.12190

Köhler, M., Haller, H., and Hartmann, E. (1999). Nuclear protein transport pathways. Nephron Exp. Nephrol. 7, 290-294. doi: 10.1159/000020616

Kosugi, S., Hasebe, M., Matsumura, N., Takashima, H., Miyamoto-Sato, E., Tomita, M., et al. (2009). Six classes of nuclear localization signals specific to different binding grooves of importin $\alpha$. J. Biol. Chem. 284, 478-485. doi: 10.1074/jbc.M807017200

Lafontaine, D. L., and Tollervey, D. (2001). The function and synthesis of ribosomes. Nat. Rev. Mol. Cell Biol. 2, 514-520. doi: 10.1038/35080045

Lange, A., McLane, L. M., Mills, R. E., Devine, S. E., and Corbett, A. H. (2010). Expanding the definition of the classical bipartite nuclear localization signal. Traffic 11, 311-323. doi: 10.1111/j.1600-0854.2009.01028.x

Lange, A., Mills, R. E., Lange, C. J., Stewart, M., Devine, S. E., and Corbett, A. H. (2007). Classical nuclear localization signals: definition, function, and interaction with importin alpha. J. Biol. Chem. 282, 5101-5105. doi: 10.1074/jbc.R600026200

Lau, C. K., Delmar, V. A., Chan, R. C., Phung, Q., Bernis, C., Fichtman, B., et al. (2009). Transportin regulates major mitotic assembly events: from spindle to nuclear pore assembly. Mol. Biol. Cell 20, 4043-4058. doi: 10.1091/mbc.E09-02-0152

Lee, B. J., Cansizoglu, A. E., Süel, K. E., Louis, T. H., Zhang, Z., and Chook, Y. M. (2006). Rules for nuclear localization sequence recognition by karyopherin $\beta 2$. Cell 126, 543-558. doi: 10.1016/j.cell.2006.05.049

Mager, W. H., Planta, R. J., Ballesta, J. G., Lee, J. C., Mizuta, K., Suzuki, K., et al. (1997). A new nomenclature for the cytoplasmic ribosomal proteins of Saccharomyces cerevisiae. Nucleic Acids Res. 25, 4872-4875. doi: 10.1093/nar/25.24.4872

Makkerh, J. P., Dingwall, C., and Laskey, R. A. (1996). Comparative mutagenesis of nuclear localization signals reveals the importance of neutral and acidic amino acids. Curr. Biol. 6, 1025-1027. doi: 10.1016/S0960-9822(02)00648-6

Marfori, M., Mynott, A., Ellis, J. J., Mehdi, A. M., Saunders, N. F., Curmi, P. M., et al. (2011). Molecular basis for specificity of nuclear import and prediction of nuclear localization. Biochim. Biophys. Acta 1813, 1562-1577. doi: 10.1016/j.bbamcr.2010.10.013

Mason, D. A., Fleming, R. J., and Goldfarb, D. S. (2002). Drosophila melanogaster importin $\alpha 1$ and $\alpha 3$ can replace importin $\alpha 2$ during spermatogenesis but not oogenesis. Genetics 161, 157-170.

Moreland, R. B., Nam, H. G., Hereford, L. M., and Fried, H. M. (1985). Identification of a nuclear localization signal of a yeast ribosomal protein. Proc. Natl. Acad. Sci. U.S.A. 82, 6561-6565. doi: 10.1073/pnas.82. 19.6561

Mosammaparast, N., Guo, Y., Shabanowitz, J., Hunt, D. F., and Pemberton, L. F. (2002). Pathways mediating the nuclear import of histones $\mathrm{H} 3$ and $\mathrm{H} 4$ in yeast. J. Biol. Chem. 277, 862-868. doi: 10.1074/jbc.M106845200

Nguyen Ba, A. N., Pogoutse, A., Provart, N., and Moses, A. M. (2009). NLStradamus: a simple Hidden Markov Model for nuclear localization signal prediction. BMC Bioinformatics 10:202. doi: 10.1186/1471-210510-202

PSORT, I. (1997). PSORT: a program for detecting sorting signals in proteins and predicting their subcellular localization. J. Mol. Biol. 266, 594-600.

Sambrook, J., and Russell, D. W. (2001). Molecular Cloning: A Laboratory Manual, $3 r d$ Edn. New York, NY: Cold Spring Harbor Laboratory Press.

Sekimoto, T., Imamoto, N., Nakajima, K., Hirano, T., and Yoneda, Y. (1997). Extracellular signal-dependent nuclear import of Stat1 is mediated by nuclear pore-targeting complex formation with NPI-1, but not Rch1. EMBO J. 16, 7067-7077. doi: 10.1093/emboj/16.23.7067

Sievers, F., Wilm, A., Dineen, D., Gibson, T. J., Karplus, K., Li, W., et al. (2011). Fast, scalable generation of high-quality protein multiple sequence alignments using Clustal Omega. Mol. Syst. Biol. 7, 539. doi: 10.1038/msb.2011.75

Soniat, M., and Chook, Y. M. (2015). Nuclear localization signals for four distinct karyopherin-beta nuclear import systems. Biochem. J. 468, 353-362. doi: 10.1042/BJ20150368

Stajich, J. E., Harris, T., Brunk, B. P., Brestelli, J., Fischer, S., Harb, O. S., et al. (2012). FungiDB: an integrated functional genomics database for fungi. Nucleic Acids Res. 40, D675-D681. doi: 10.1093/nar/gkr918 
Süel, K. E., and Chook, Y. M. (2009). Kap104p imports the PY-NLS-containing transcription factor Tfg2p into the nucleus. J. Biol. Chem. 284, 15416-15424. doi: 10.1074/jbc.M809384200

Süel, K. E., Gu, H., and Chook, Y. M. (2008). Modular organization and combinatorial energetics of proline-tyrosine nuclear localization signals. PLoS Biol. 6:e137. doi: 10.1371/journal.pbio.0060137

Timmers, A. C., Stuger, R., Schaap, P. J., van 't Riet, J., and Raue, H. A. (1999). Nuclear and nucleolar localization of Saccharomyces cerevisiae ribosomal proteins S22 and S25. FEBS Lett. 452, 335-340. doi: 10.1016/S0014-5793(99)00669-9

Truant, R., Kang, Y., and Cullen, B. R. (1999). The human tap nuclear RNA export factor contains a novel transportin-dependent nuclear localization signal that lacks nuclear export signal function. J. Biol. Chem. 274, 32167-32171. doi: $10.1074 /$ jbc.274.45.32167

Tyler, B. M. (2007). Phytophthora sojae: root rot pathogen of soybean and model oomycete. Mol. Plant Pathol. 8, 1-8. doi: 10.1111/j.1364-3703.2006.00373.x

Underwood, M. R., and Fried, H. M. (1990). Characterization of nuclear localizing sequences derived from yeast ribosomal protein L29. EMBO J 9, 91.
Xu, D., Farmer, A., and Chook, Y. M. (2010). Recognition of nuclear targeting signals by Karyopherin- $\beta$ proteins. Curr. Opin. Struct. Biol. 20, 782-790. doi: 10.1016/j.sbi.2010.09.008

Zhang, M., Lu, J., Tao, K., Ye, W., Li, A., Liu, X., et al. (2012). A Myb transcription factor of Phytophthora sojae, regulated by MAP kinase PsSAK1, is required for zoospore development. PLoS ONE 7:e40246. doi: 10.1371/journal.pone.0040246

Conflict of Interest Statement: The authors declare that the research was conducted in the absence of any commercial or financial relationships that could be construed as a potential conflict of interest.

Copyright (C) 2017 Fang, Jang, Watson, Wellappili and Tyler. This is an open-access article distributed under the terms of the Creative Commons Attribution License (CC $B Y)$. The use, distribution or reproduction in other forums is permitted, provided the original author(s) or licensor are credited and that the original publication in this journal is cited, in accordance with accepted academic practice. No use, distribution or reproduction is permitted which does not comply with these terms. 